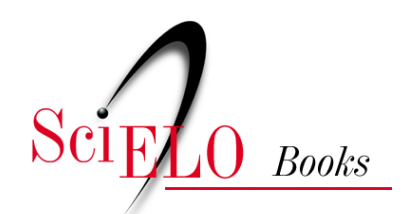

\title{
Capítulo I - Óptima planeación de recursos para redes eléctricas inteligentes usando modelos heurísticos
}

\author{
Esteban Inga \\ Miguel Campaña
}

\section{SciELO Books / SciELO Livros / SciELO Libros}

INGA, E., and CAMPAÑA, M. Óptima planeación de recursos para redes eléctricas inteligentes usando modelos heurísticos. In: INGA ORTEGA, E., ed. Aplicaciones e innovación de la ingeniería en ciencia y tecnología [online]. Quito: Editorial Abya-Yala, 2019, pp. 11-56. ISBN: 978-9978-10491-0. https://doi.org/10.7476/9789978104910.0003.

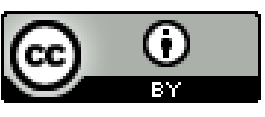

All the contents of this work, except where otherwise noted, is licensed under a Creative Commons Attribution 4.0 $\underline{\text { International license. }}$

Todo o conteúdo deste trabalho, exceto quando houver ressalva, é publicado sob a licença Creative Commons Atribição 4.0.

Todo el contenido de esta obra, excepto donde se indique lo contrario, está bajo licencia de la licencia $\underline{\text { Creative }}$ Commons Reconocimento 4.0 . 


\title{
Capítulo I
}

\section{Óptima planeación de recursos para redes eléctricas inteligentes usando modelos heurísticos}

\section{Optimal Planning of Resources for Smart Grid using Heuristic Methods}

\author{
Esteban Inga*, Miguel Campaña ${ }^{\S}$ \\ Universidad Politécnica Salesiana Ecuador \\ *Email: einga@ups.edu.ec \\ §Email: mcampana@ups.edu.ec
}

\section{Resumen}

El presente trabajo considera una planificación de redes inalámbricas heterogéneas para proporcionar medición inteligente dentro de una red de área vecina con su respectiva conectividad con la oficina central de una compañía de distribución eléctrica. Además, el trabajo presenta un modelo de planificación para lograr una red de distribución eléctrica subterránea en un área definida. Tanto la red inalámbrica heterogénea como la red de distribución se implementan en un área georeferenciada real y permiten minimizar el costo por el uso de recursos de telecomunicaciones y eléctricos; de esta forma, se logra el concepto de una red eléctrica inteligente eficiente al minimizar los costos y aumentar la confiabilidad de la red considerando las restricciones de capacidad y la cobertura del usuario.

\section{Palabras claves}

Sistemas eléctrico de distribución; optimización; planeación; medición inteligente; redes eléctricas inteligentes

\section{Abstract}

The present work considers a planning of heterogeneous wireless networks to provide smart metering within a neighborhood area network with its respective connectivity with the central office of an electricity distribution company. In addition, a planning model is present to achieve an underground electrical distribution network in a defined area. Both 
the wireless heterogeneous network and the distribution network are deployment over a real georeferenced area and allow minimizing the cost by use of telecommunications and electrical resources; in this way, the concept of an efficient intelligent electric network is meet by minimizing costs and increasing reliability of the network considering capacity restrictions and user coverage.

\section{Keywords}

Electrical distribution system; optimization; planning; smart metering; smart grid.

\section{Introducción}

Para facilitar la conversión de una red eléctrica convencional a una red eléctrica inteligente es necesario incluir un sistema de comunicaciones que permitan el intercambio de información bidireccional [1],[2]. El presente trabajo se enfoca en la infraestructura de medición inteligente de energía eléctrica; para lo cual, se requiere la conectividad entre el medidor inteligente de cada usuario y la oficina central de una empresa eléctrica, logrando una medición inteligente del suministro eléctrico que se provee a cada usuario o cliente por parte de una empresa de distribución eléctrica [3].

La infraestructura de medición inteligente podrá estar formada de varias subredes con diferentes tecnologías de comunicación para cada caso, así tenemos que la red de área doméstica estará dedicada al intercambio de información entre el medidor inteligente y los sensores ubicados en el interior de la vivienda, esta información podrá ser utilizada para caracterizar el consumo de cada usuario de manera particular, en este punto, un detalle importante se relaciona a la seguridad de la información, que aunque no está involucrado en el presente trabajo, debe preocupar a las empresas eléctricas y a los usuarios, ya que, podría sufrir ataques de parte de la delincuencia y con este conocer el estado y hasta el número de dispositivos electrónicos que posee un cliente, tiempo de uso, tarifa de pago, etc., [4], [5].

A continuación, se desprende la red de área vecindaria, pilar fundamental de este trabajo, donde se encuentra la mayor necesidad de optimizar los recursos, ya que, los usuarios podrán estar ubicados en zonas urbanas con mayor cercanía entre una red de comunicaciones; existente en relación con una zona suburbana, pero también estarán las zonas rurales con baja penetración de la 
red de comunicaciones y requerirá que los recursos de tecnología de comunicaciones sea la idónea. En todos los casos anteriores se deberá tener una planeación adecuada que permita una rápida instalación de medidores inteligentes, pero de forma escalable; de esta manera se garantiza que el costo por recursos sea el mínimo. Los criterios de partida para iniciar el despliegue no solo podrán depender de atacar zonas donde existe mayor robo o hurto de energía eléctrica, sino que, a qué tipo de zona se refiere y qué característica en disponibilidad de tecnología de comunicaciones existe para determinar el hardware que deberá disponer un medidor inteligente. De lo antes mencionado, es prescindible resaltar que una única solución de comunicaciones no es viable, sino que, puede existir múltiples soluciones y con el menor costo [6],[7].

La red de área amplia encargada de la conectividad entre los medidores inteligentes con la empresa de distribución eléctrica pasa a formar una zona de frontera debido al requerimiento de concentradores que aglutinarán información proveniente de un conjunto de medidores inteligentes y a su vez permite el intercambio de información o enlace de última milla con una estación base celular, elemento que finalmente enlaza o envía información con la oficina central [8].

Tal como se aprecia el envío de información desde un medidor inteligente a una empresa de distribución eléctrica toma el nombre de enlace ascendente, pero se debe tener presente que la información puede ser descendente debido a que la medición inteligente también permite cortes y reconexiones del suministro eléctrico con lo que a través de la información recibida se activa o desactiva el sistema electrónico del medidor inteligente y así se permite o no el paso del suministro eléctrico a la vivienda del cliente [9],[10].Como complemento a la planeación de la red de comunicaciones para medición inteligente es primordial dotar de un modelo que permita, en la misma zona georreferenciada, el dimensionamiento de una red eléctrica capaz de proporcionar mapas de rutas óptimos con la finalidad de construir redes eléctricas, ya sean: soterradas, aéreas o en su defecto mixtas muy pertinentes para facilitar que la red eléctrica inteligente consiga confiabilidad y calidad del suministro [11]-[15].

La creciente demanda por el incremento de cargas significativas a la red eléctrica de distribución existente, como: introducción 
de cocinas de inducción, calentamiento de agua, incremento de nuevos usuarios a la red eléctrica o por la integración de vehículos eléctricos en un inmediato próximo [16] hace que, la necesidad de redimensionar equipos eléctricos de potencia reconsiderando capacidades nominales de corriente en los conductores y otras variables eléctricas sensibles al incremento de la demanda sean nuevamente analizados para garantizar un servicio eléctrico de calidad y confiable [17],[18]. En redes eléctricas nuevas, se debe planificar considerando las demandas tipo actualizadas que contemplan la energía demandada por cocción y calentamiento de agua. Por lo tanto, se propone implementar una heurística capaz de solucionar el problema de planeación en redes eléctricas de distribución considerando restricciones de cobertura lineal y capacidad de albergar usuarios en los transformadores de distribución a emplazarse en sitios georreferenciados, determinados por la heurística, garantizando satisfacer el máximo porcentaje de observabilidad a los usuarios sin descuidar los principios técnicos de calidad y seguridad energética. El modelo será implementado en el software Matlab y mediante el software Cymdist se analizará el flujo de potencia resultante obtenido del mapa de ruta lograda por la heurística propuesta.

El modelo de planeación de redes de distribución es capaz de proporcionar datos de mucho interés para iniciar un despliegue óptimo en una red eléctrica de distribución nueva, tales como: cantidad de transformadores por cada capacidad en kVAs requerido en el despliegue, caídas de voltaje máximos en cada transformador emplazado y capacidad máxima requerida en la subestación para satisfacer la demanda total. Además, el modelo admite parámetros reales de diseño, tales como: kVA-m soportado por el conductor a utilizarse en bajo voltaje, demandas por cada usuario identificando a qué estrato pertenece, estandarización de transformadores y distancia promedio en la que se debe emplazar los pozos en una red eléctrica soterrada o los postes en una red eléctrica aérea. El modelo da solución al problema de planeación aplicando teoría de grafos y enrutamiento de mínima y máxima expansión [19], garantizando así, proporcionar topologías al menor costo.

El análisis eléctrico obtenido del software Cymdist, tomado en cuenta, el despliegue y datos obtenidos por la heurística propuesta conocido en la literatura como modelo de red de referencial [20], 
contribuirá de manera óptima a la toma de decisiones garantizando una red eléctrica segura y resiliente al menor costo de implementación. El algoritmo de planeación y despliegue de una red eléctrica de distribución consta de cinco procedimientos. El primer procedimiento consiste en levantar la información necesaria georreferenciada de un archivo OSM obtenido de OpenStreetMap, el mismo que, contiene la información necesaria de las coordenadas (latitud y longitud) de calles, viviendas, parques, etc.

En el segundo procedimiento se genera múltiples escenarios de conectividad observando las restricciones anteriormente mencionadas con técnicas de clusterización basado en una variante del algoritmo de PRIM. Mediante el algoritmo del Setcover se procede, en el paso tres, a seleccionar el mínimo número de subconjuntos de conectividad que contengan el mayor número de elementos, para así, proporcionar una solución cercana a la óptima.

En consecuencia, la solución obtenida por la heurística, no se basa en la topología de una red inicial, sino que, se basa en la conjugación de varias soluciones, de tal manera que, se busca garantizar una solución óptima global.

En el procedimiento cuatro, se eliminan elementos redundantes de los subconjuntos seleccionados por el algoritmo Setcover, posteriormente, de cada subconjunto o agrupación, bajo el criterio del centro de masa, se selecciona la ubicación del transformador a emplazarse y se procede a construir un árbol de mínima expansión, mediante el algoritmo de Dijkstra entre los primarios de los transformadores habilitados dando origen a la red de medio voltaje y el mismo criterio de aplica en los secundarios, con cada agrupación, para formar la red de bajo voltaje, a diferencia que, se emplea un árbol de máxima expansión con la finalidad de considerar a todas las viviendas de cada subconjunto para el tendido eléctrico desde los secundarios de los transformadores de distribución hacia sus nodos de servicio más lejanos. Por último, se procede a calcular las caídas de voltaje en cada transformador emplazado (desde la fuente hacia el nodo más lejano).

En adelante este artículo se organiza de la siguiente manera. En la sección 2 se introduce el modelo de planeación para redes de comunicaciones inteligente y para redes eléctricas de distribución. En la sección 3 se describe la formulación del problema para una red 
heterogénea inalámbrica y la red eléctrica de distribución soterrada. En la sección 4 se analizan los resultados de la modelización y simulación de los algoritmos de planeación. Finalmente, concluimos este artículo en la sección 5 .

\section{Planeación Óptima de Redes}

\section{A. Red heterogénea inalámbrica para medición inteligente}

Si consideramos medidores inteligentes de energía eléctrica con intervalos de lectura cada 15 minutos, en 24 horas tendríamos 96 lecturas por día y en un mes 2880 lecturas. Por otro lado, si se considera que cada lectura representa 5 bytes; en un escenario de 4’000.000 de abonados, al multiplicar por 5 bytes nos genera una cantidad aproximada a 20 '000.000 bytes por lectura. La Tabla 1, muestra el crecimiento de la información para un intervalo entre cada lectura [21],[11], [22]. El concepto de los recursos requeridos por la infraestructura de medición avanzada se avizora en la Figura 1, de acuerdo a este modelo se incorporan concentradores o puntos de agregación de datos (PAD) que permitirán la conectividad con las estaciones base celular y de esta manera se reducen los costos al incorporar tecnología inalámbrica con un costo menor a la tecnología celular.

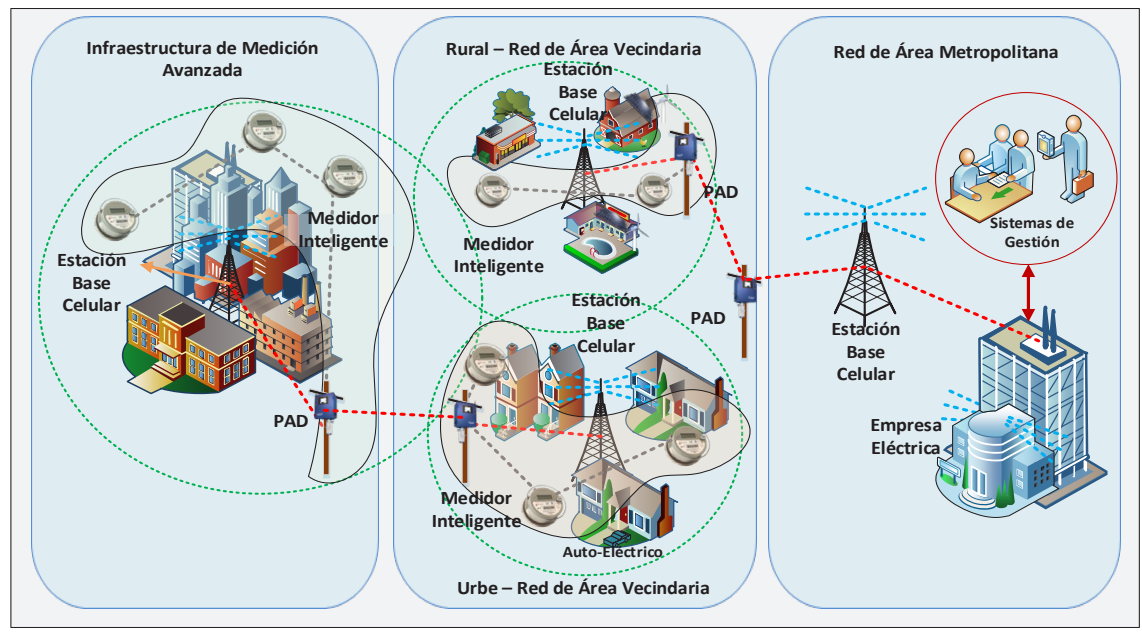

Figura 1. Infraestructura de medición avanzada usando redes inalámbricas 
Tabla 1. Cantidad de información para un intervalo de lectura de $\mathbf{1 5}$ minutos

\begin{tabular}{|c|c|c|c|c|}
\hline $\begin{array}{c}\text { Número de } \\
\text { Medidores }\end{array}$ & Datos & Lecturas & $\begin{array}{c}\text { Intervalo de } \\
\text { Tiempo }\end{array}$ & Cantidad de Datos \\
\hline $4 \mathrm{M}$ & 5 Bytes & 96 & 1 Día & $1.92 \mathrm{~GB}$ \\
\hline $4 \mathrm{M}$ & 5 Bytes & 96 & 1 Mes & $57.6 \mathrm{~GB}$ \\
\hline $4 \mathrm{M}$ & 5 Bytes & 96 & 1 Año & $691.2 \mathrm{~GB}$ \\
\hline
\end{tabular}

De los datos preliminares se puede apreciar que el crecimiento de la información es considerable, más aún si se requieren obtener reportes de esa cantidad de datos de manera eficiente para una posterior toma de decisiones. Es así que, lograr una infraestructura de medición avanzada, resulta importante investigar para lograr minimizar el impacto que esta red dispondría para una empresa de distribución eléctrica.

Para crear una infraestructura de medición avanzada se han realizado varios aportes científicos que han buscado dar solución desde diferentes frentes y así conseguir una medición inteligente de energía eléctrica; sin embargo, las restricciones propias de la tecnología, las zonas, el costo, hacen de la planeación de la red de comunicaciones un problema no trivial que en términos de optimización se describe como un problema NP-Complete dentro de los capítulos propios de problemas combinatorios; por lo tanto, conseguir una única solución que nos entregue el óptimo global de la planeación de la red de comunicaciones resulta inviable; sin embargo, es posible advertir que las tecnologías se encuentran maduras y/o ya desplegadas con el objeto de minimizar el costo y garantizar la confiabilidad de la red [2], [5], [23]-[25].

En sus inicios para conseguir una medición inteligente se avizoraba un crecimiento oportuno para una solución con radio frecuencia ya sea en el rango de $902-928 \mathrm{MHz}$ o en $169 \mathrm{MHz}$, en este sentido, aunque no existía costo por uso de frecuencia sí se requería un número considerable de concentradores con RFID; finalmente esta solución no dio eficiencia en el despliegue debido a que la configuración y mantenimiento de los recursos los debía absorber las empresas de distribución eléctrica destinadas tradicionalmente a un trabajo diferente por la naturaleza de su negocio. En un camino por solventar el problema de cobertura y permitir la bidireccionalidad de la red, se 
realizaron enlaces de última milla colocando una tarjeta de comunicación con tecnología celular que permita el enlace con las estaciones base celular más cercanas.

Posterior a los inicios de RFID orientado a disminuir las pérdidas no técnicas de energía proveniente del robo o hurto de clientes residenciales, se advirtió una posibilidad para la medición inteligente de energía eléctrica de clientes industriales o especiales ubicados de manera aislada pero dentro de zonas con cobertura celular, dando origen a una solución única con tecnología celular, este costo por los recursos y el servicio del operador primario de telefonía celular al ser un escenario pequeño no presentaba dificultad y se generaron dichos despliegues con satisfacción compartida, tanto para el cliente industrial,como para la empresa de distribución que entrega el servicio eléctrico.

Actualmente, operadoras de telefonía celular han intentado dar servicio de medición inteligente incluyendo opciones para energía eléctrica, agua y gas, basándose en que únicamente cambia el medidor inteligente y han realizado pruebas NB-IoT que utilizan bandas celulares y que nació tradicionalmente como una tecnología de radio de banda estrecha para aplicaciones de internet de las cosas, por otro lado, se tiene el uso del ahora estándar IEEE 802.15.4g que tiene un costo menor a WiFi y aunque ha mejorado el alcance no ha llegado a destacar en aplicaciones de medición inteligente.

Finalmente, y con mayor penetración en soluciones de medición inteligente de energía eléctrica se encuentra WiFi utilizada en exteriores que puede lograr conectividad entre concentradores y medidores inteligentes; por lo tanto, puede convertirse en una fuerte opción para lograr una planeación escalable de medición inteligentes de energía eléctrica, agua y gas. Para lograr que esta solución sea factible de implementar se debe tener en cuenta que al ser una solución híbrida se permite acoplar varias tecnologías, en este caso, de índole inalámbrico para formar una red heterogénea la misma que brinda la posibilidad de realizar multi saltos entre medidores inteligentes en zonas urbanas donde existen viviendas circundantes en un radio pequeño y en zonas suburbanas y rurales donde no exista posibilidad de multi salto entre el hardware de comunicación de cada medidor inteligente [26],[27]-[31]. 


\section{B. Red de distribución eléctrica soterrada}

La necesidad de actualización de las redes eléctricas de distribución debido a la creciente penetración de recursos energéticos distribuidos, junto con el crecimiento de la carga, eficiencia energética, seguridad y calidad del suministro eléctrico han cambiado de forma significativa los enfoques tradicionales de planeación, diseño y operación del sistema de energía eléctrica [32]. Para superar los desafíos operacionales del sistema eléctrico de distribución se han empleado un gran número de algoritmos, siendo implementados en unas pocas redes específicas de prueba que aún no son capaces de proporcionar conclusiones sólidas y generalizadas [33],[34],[35].

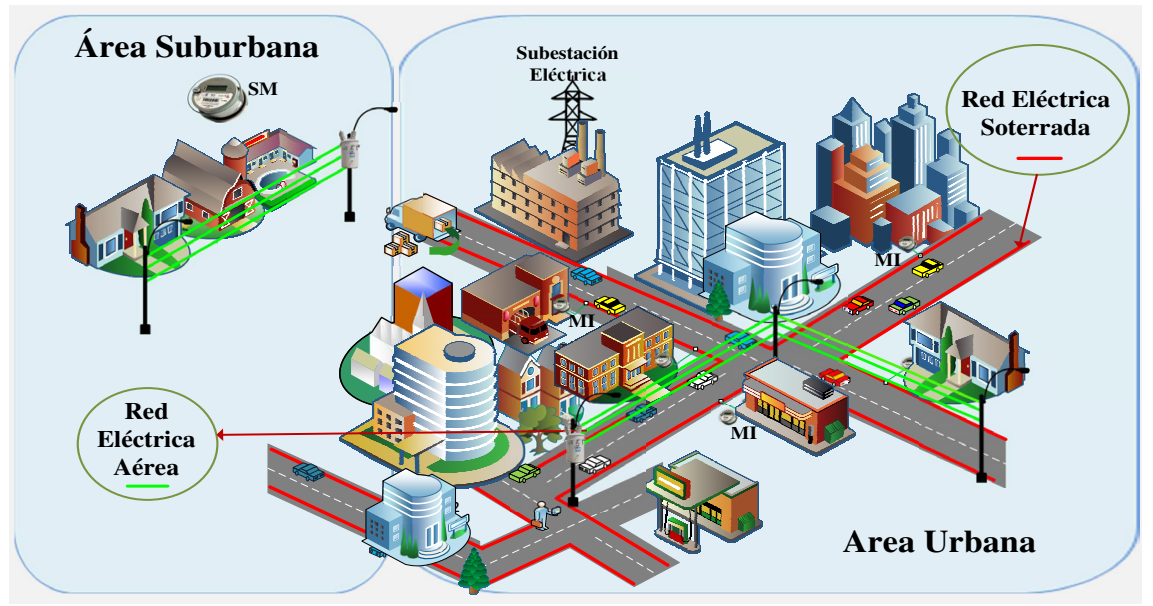

Figura 2. Planeación de redes eléctricas de distribución considerando arquitecturas soterradas y aéreas

En el presente documento se analiza desde dos enfoques realistas que son propiedades intrínsecas en los sistemas eléctricos de distribución, los cuales son: propiedades topológicas y propiedades eléctricas. Ambas propiedades eléctricas y topológicas de redes eléctricas de distribución tienen un impacto significativo en el rendimiento global de la red incluyendo caídas de tensión, pérdidas de potencia, fiabilidad de la red y, por último, cada impacto se ve reflejado en los costos. Por lo tanto, la presente investigación se centra en la planificación de redes eléctricas de distribución considerando restricciones reales con 
la finalidad de proporcionar topologías seguras para la implementación al menor costo como se ilustra en la Figura 2 y, por último, en un segundo escenario, mediante el software Cymdist, trabajar sobre la segunda propiedad de una red eléctrica de distribución.

Con la finalidad de comprender y modelar una red compleja real soterrada y con la posibilidad de ser aérea o, en su defecto mixta, un conjunto de herramientas y técnicas son necesarias. En consecuencia, estas técnicas y herramientas tienen que ver con análisis de redes complejas y teoría de grafos. La red eléctrica soterrada dispone de varios elementos tales como líneas de transmisión, transformadores de distribución, pozos, zanjas y dispositivos de protecciones y maniobra eléctrica. Un modelo gráfico puede ser fácilmente construido teniendo en cuenta la relación de cada uno de los elementos existentes en la red eléctrica a desplegarse.

Para poder construir redes eléctricas de distribución es de mucha importancia considerar definiciones que se utilizan en la investigación topológica, tales como: propiedades de un grafo, propiedad de profundidad y propiedad topológica en redes eléctricas de distribución considerando coordenadas georreferenciadas.

Un grafo está formado por vértices $(V)$ y aristas $(E)$, donde, su expresión formal es $G=(V, E)$. En redes eléctricas de distribución, los vértices están asociados a los trasformadores de distribución, subestaciones, usuarios, interruptores, barras colectoras y pozos (redes soterradas) o postes (redes aéreas). La información de cada elemento, en relación con su posición, está dada en coordenadas geolocalizadas (Latitud y Longitud), donde, $i$ representa el vértice de origen y $j$ el vértice destino, que, mediante una arista $E$ ambos vértices se relacionan. Al gráfico de conectividad se lo representa con una matriz de adyacencia cuadrada nxn, la misma que, relaciona a todos los vértices con sus respectivas aristas de manera controlada, es decir, sujeta a restricciones. Dichas restricciones en el presente documento son distancias lineales y capacidad de los trasformadores de distribución de albergar usuarios. Por lo tanto, la matriz cuadrada de conectividad es una matriz binaria, donde, 1 representa la existencia de una arista de un nodo $v i$ a un $v j$ y, por el contrario, 0 representa la no conectividad o la no existencia de una arista de un $v i$ a un $v j$. En consecuencia, la matriz de conectividad se irá llenando de valores binarios coherentemente con las restriccio- 
nes consideradas en el modelo para dar solución a la planeación de redes eléctricas de distribución. Por último, dentro de las propiedades del grafo, se considera, la longitud de la red que está dada por las distancias acumuladas o parciales de las aristas, además se considera la agrupación de los nodos que están directamente asociados con la capacidad del transformador de distribución.

En la propiedad de profundidad se analiza la topología armada por el grafo no dirigido $G$ desde la fuente hacia los usuarios finales, el mismo que, en redes eléctricas de distribución en los niveles de medio y bajo voltaje son radiales o tipo árbol [32]. Un grafo con topología tipo árbol está definido por la utilización mínima de aristas para la conexión de todos sus vértices, es decir, si es el número de nodos o usuarios un árbol relaciona a todos sus vértices con $n$ - 1 aristas. En el presente documento se genera la ruta al mínimo costo utilizando el algoritmo de Dijkstra en la red de medio voltaje y mediante una breve modificación se genera el árbol de máximo costo para la red de bajo voltaje. En la red de bajo voltaje se maximiza, puesto que, es necesario satisfacer de cobertura a todos los usuarios relacionados con sus respectivos subconjuntos.

En las propiedades topológicas de la red de distribución se observa la realidad del escenario, en el que, se contempla la cantidad de nodos por $\mathrm{km}^{2}$, longitud de la red en medio voltaje requerido por $\mathrm{km}^{2}$, número de alimentadores y número de pozos en el caso de despliegues soterrados, son variables que, claramente caracterizan las diferencias topológicas entre tipos de redes aéreas o soterradas. Otro elemento determinante para la topología de la red eléctrica es la técnica aplicada de clusterización, la misma que, agrupa elementos de objetos similares. En la presente investigación empleamos para dicho cometido, una variante del algoritmo de PRIM, sin considerar, el conocimiento de la cantidad de agrupaciones existentes, es decir, el modelo determina el número óptimo de agrupaciones para garantizar el máximo número de usuarios cubiertos por la red eléctrica.

\section{Formulación del problema}

Esta sección está dividida en dos partes: a) describe las variables que describen el problema para la planeación de una red heterogénea inalámbrica dedicada a la medición inteligente de energía eléctrica y b) 
las variables que se involucran para resolver el problema de ubicación de transformadores en una red de distribución eléctrica soterrada.

\section{A. Red heterogénea inalámbrica}

Si partimos de formalismos matemáticos, el problema de ubicación óptima de PADs en una red de área vecindaria se la puede describir desde el concepto de teoría de grafos como un grafo no dirigido, el mismo que describe en un conjunto de vértices y como los enlaces, en este trabajo los medidores inteligentes se encuentran representados por los vértices y la red inalámbricas por los enlaces.

El escenario propuesto plantea tres elementos o recursos, estaciones base celular, PADs y MIs; para ello, es necesario tener en cuenta que un MI puede enviar información a un PAD o a otro MI lo que permite que a través de multi salto se envíe información hasta un PAD. Existe conectividad entre PADs para lograr conectividad con la estación base celular más cercana que finalmente realizar el trabajo de última milla con la empresa de distribución eléctrica.

Para el escenario propuesto los enlaces dentro del grafo no dirigido representan el costo determinado para este caso por la distancia, entonces se consideran únicamente valores positivos. Se supone que la tecnología inalámbrica a ser utilizada tanto en los MIs como en PAD es flexible, pero para el escenario se considera una red WiFi y celular. De esta manera el radio de transmisión puede ajustarse a las necesidades de la zona de despliegue, determinadas por las zonas urbanas, suburbanas o rurales; entonces, no todos los MIs tienen una única tecnología de comunicación. El alcance de comunicación de cada MI lo describe la variable ; además, definamos un conjunto $U=\{s 1, s 2, \ldots s N\}$, de MIs. La posición del MI i-ésimo está dada por $\left(x s_{i}, y s_{i}\right)$, donde $x s i, y s i \in S$.. El MI de coordenadas $x s i, y s i$ puede conectarse a otro MI de coordenadas $x s j$,ysj $s$ si la distancia es menor o igual a distancia permitida $\left|\left(x s_{i} y s_{i}\right)-\left(x s_{j} y s_{j}\right)\right| \leq d m i n$. Por lo tanto, se tiene un subconjunto $S_{d m i n}$ (xui,yui), así todo MI que tenga alcance en el rango de la tecnología inalámbrica propuesta está dada por $S_{d \min }\left(x u_{p} y u_{i}\right)=\left|\left(x s_{p} y s_{i}\right)-\left(x s_{p} y s_{j}\right)\right| \leq d \min , \forall_{j} \in S$

En caso de que un MI no esté en el rango de conectividad de otro MI podrá conectarse a un $\mathrm{PAD}$, para este caso se ha restringido la posibilidad que un MI pueda conectarse directamente a una esta- 
ción base celular con el objeto de reducir los costos; sin embargo, un MI puede quedar sin conectividad ya sea por capacidad de MI más cercano o del PAD, pero de estar en el radio de alcance de una estación base celular puede ser conectado por tecnología celular directamente. Es importante recordar que un MI en su arquitectura tiene la flexibilidad de contar con un módulo de tecnología inalámbrica WiFi, IEEE 802.15.4g, Celular, etc. Así, tenemos un grafo que garantiza la conectividad de los tres recursos inicialmente expuestos.

Para que un modelo de planeación sea considerado para uso real en las acciones de despliegue por parte de una empresa de distribución eléctrica es necesario contar con coordenadas georreferenciadas de latitud y longitud; además, de un cálculo de la distancia con la fórmula de haversine que indica la ecuación 1 , fórmula que considera la curvatura de la tierra. De esta manera $x s_{j}, y s_{i}$ es la longitud y latitud de los MIs

Distancia $=2 * R * \operatorname{asin}^{2} \sqrt{\sin ^{2}\left(\frac{\Delta l a t}{2}\right)}+\cos ($ lat 1$) * \cos ($ lat 2$) * \sin ^{2}\left(\frac{\Delta l o n}{2}\right)$

Para aplicar la ecuación 1 se debe considerar a lat como la latitud y lon como la longitud; $\Delta$ lat es la diferencia de; lat 2 - lat $1 ; \Delta l o n=$ lon 2 - lon $1 ; \mathrm{R}=6372.795477598 \mathrm{~km}$ que es el radio de la tierra.

El trabajo propuesto para generar el modelo de planeación escalable no debe ser planteado como una simple partición del grafo inicialmente determinado por $G=(V, E)$ y subdividido en conjuntos o subredes más pequeñas a través de una clusterización en la que se puede configurar una capacidad para cada centroide que entregaría las coordenadas de un PAD y la razón se debe a que los métodos de clusterización nos entregan un resultado diferente y con subconjunto desbalanceados en lo que se refiere al número de MIs por cada PAD.

La variable es determinante para el proceso de enrutamiento; por tal razón, se requiere encontrar la distancia más corta no solo entre cada MI sino también con cada PAD ya que en el despliegue de una red heterogénea los multi saltos no deben ser muy largos para evitar retardos en el envío de paquetes o mayor consumo de energía.

A continuación, se detalla el algoritmo utilizado en el modelo OPDWHN-AMI: 


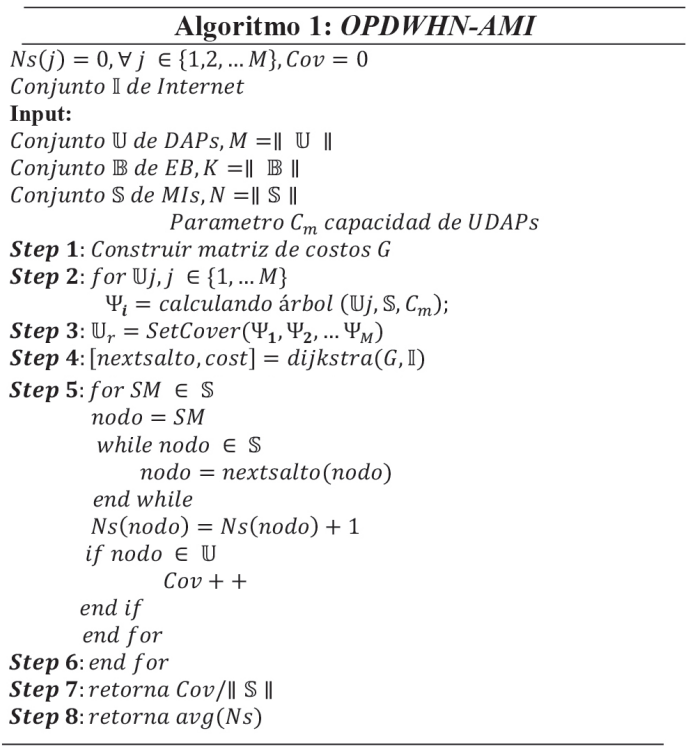

La función $\Psi$ implementada como un método recursivo de la heurística OPDWHN-AMI se presenta a continuación:

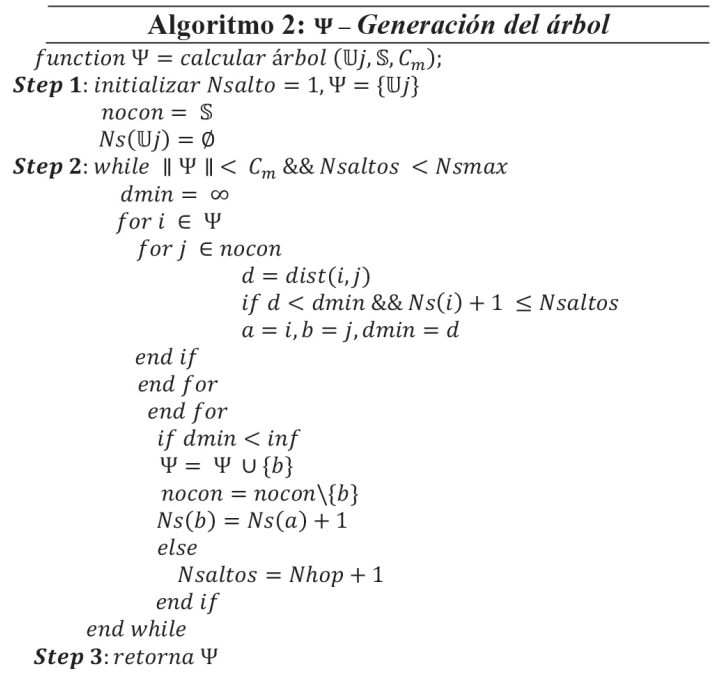


El modelo de planeación de redes heterogéneas inalámbricas bajo las consideraciones anteriormente descritas define un problema combinatorio del tipo NP-Complete; por lo tanto, requiere un proceso heurístico para obtener un resultado cercano al óptimo que permita en la línea del tiempo seguir incluyendo nuevos MIs y PADs de ser el caso.

Mediante la tabla 2 se hace un resumen de las variables empleadas en el modelo de redes inalámbricas heterogéneas.

\section{B. Red eléctrica soterrada}

El presente modelo para planeación de redes de distribución eléctrica soterrada considera similar principio al aplicado para medición inteligente, con una variante en relación a la restricción para no permitir enlaces de cobertura radiales, sino que, permitir enlaces de cobertura lineales, evitando así, diagonales dentro del $G=(V, E)$ $y$, en consecuencia, se logra construir enlaces controlados sin cruces por los espacios delimitados por calles en todos sus lados, de esta manera, el despliegue de la red soterrada deberá seguir únicamente el camino preestablecido que refiere a calles y avenidas, en este caso, el problema advierte la necesidad ubicar de manera óptima el número de transformadores de distribución eléctrica; así como, el número de pozos de revisión que se requieran para el despliegue; para lo cual, se tomará información bajo normativas y estándares específicos para redes soterradas.

Para resolver el problema combinatorio definido como NP-Complete, planteado en este trabajo, se incluye un modelo heurístico que permite encontrar el costo mínimo para lograr el máximo porcentaje de cobertura de servicio para los usuarios finales. A continuación, en la ecuación 2 se presenta el costo total de cada transformador perteneciente a cada clúster, donde, es el número de elementos de cada clúster y $C_{p}$ es la demanda individual de cada usuario dependiendo de la demanda estratificada tipo al que pertenezca. En la ecuación $3, C_{\text {sub }}$ es el costo total de la subestación, donde, es el número de clústeres existentes en el escenario. Un aspecto de mucha importancia es que, en cada iteración para obtener el costo de la subestación, se obtiene el costo acumulado por el algoritmo en cada transformador de distribución a desplegarse en el área de interés. De aquí en adelante, entiéndase por costo a los valores en MVAs o 
kVAs (transformadores, subestaciones) y longitudes (conductores), según

$$
\begin{gathered}
C_{t r}=\sum_{k 1=1}^{z e} C_{p}(k 1), \quad \forall z e, C_{p} \in \mathbb{R}^{+}>0 ; \\
C_{s u b}=\sum_{k 1=1}^{z e} \sum_{p=1}^{l e} C_{t r}(k 1, p), \quad \forall z e, l e \in \mathbb{R}^{+}>0 ;
\end{gathered}
$$

En la ecuación 4, el costo total del conductor en la red de medio voltaje está representado por $C m v_{\text {con }}$, la expresión $d_{a}$ es la longitud del tramo de un punto $i$ a un punto $j$, el factor de multiplicación $k$ es una variable de costo por cada metro para la implementación de la red de medio voltaje y, por último, $N$ es la longitud del vector que contiene las posiciones de los transformadores incluyendo la posición de la subestación. Por otro lado, la expresión $N$-1 representa el número de aristas mínimo para garantizar de conectividad a todos los transformadores desde la subestación.

$$
C m v_{\text {con }}=\sum_{a=1}^{N-1} d_{a} * k, \quad \forall N \in \mathbb{R}^{+}>1 ;
$$

En la ecuación 5, se presenta el costo total del conductor de la red de bajo voltaje multiplicado por el factor $K$ explicado anteriormente. La expresión $d_{(h, a)}$ representa las distancias máximas requeridas en cada tramo para garantizar la conectividad desde la fuente hacia los nodos de servicio, la misma que, representa a la distancia más crítica, es decir, existirá distancias menores dentro de cada agrupación, pero no mayores.

$$
C b v_{\text {con }}=\sum_{h=1}^{l e} \max \sum_{a=1}^{z i s e(z e)-1} d_{(h, a)} * k, \quad \forall d \in \mathbb{R}^{+} ;
$$

En consecuencia, el problema de optimización, para planeación de redes eléctricas de distribución, se define de la siguiente manera mediante la ecuación 6 :

$$
\min C_{t r}+C_{s u b}+C m v_{c o n}+C b v_{c o n}
$$

Sujeto a: 


$$
\begin{gathered}
\sum_{\phi_{i} \in \phi} \phi \leq \tau, \quad \forall \phi \in A(\beta) ; \forall \tau>1 \\
\sum_{i=1}^{\Omega} \phi_{i}=\beta, \quad \forall i \in A(\beta) ; \forall \Omega \in \mathbb{R}^{+} \\
d_{i, j} \leq \lambda, \quad \forall i, j \in A(\beta)
\end{gathered}
$$

La ecuación 7, se expresa la restricción de capacidad, con lo cual, se limita el número de usuarios asociados a un transformador de distribución, donde, $\tau$ es la cantidad de elementos intra-clúster máximo permitido, $A(\beta)$ es el conjunto de usuarios emplazados en el área de interés y $\phi$ son los subconjuntos de agrupaciones. La ecuación 8 , es una rutina de comprobación, en la cual, se verifica que la suma de los nodos de cada agrupación sea igual a la longitud de $\beta$ de usuarios desplegados en el área. De esta manera, se garantiza que los subconjuntos creados cubran el 100\% de los usuarios emplazados en el área de estudio. Con la ecuación 9 se garantiza que, en cada agrupación, únicamente existan elementos que cumplan con la restricción de distancia máxima permitida para asociar a un usuario

\begin{tabular}{|c|c|c|}
\hline Símbolo & Descripción & Significado \\
\hline II & Internet & Conjunto internet \\
\hline $\mathbb{B}$ & Estación base cellular & Conjunto EB \\
\hline $\mathbb{U}$ & & Conjunto PADs \\
\hline $\mathbb{S}$ & Medidores Inteligentes & Conjunto de MIs \\
\hline$C_{m}$ & Capacidad & Capacidad PAD \\
\hline $\mathrm{G}$ & Matriz de conectividad & Matriz de costo \\
\hline dist & distancia & $\begin{array}{l}\text { Distancia haversine entre } \\
\text { recursos }\end{array}$ \\
\hline Cov & Cobertura & Cobertura de MIs \\
\hline$\Psi$ & Arbl & Steiner tree \\
\hline $\mathbb{U}_{r}$ & Set cover & Set cover/ radio de cobertura \\
\hline Ns & MIs conectados & Promedio de MIs conectados \\
\hline$\Psi_{i}$ & Conjunto de MIs $\rightarrow$ PAD & Direct $\mid$ multi saltos \\
\hline nocon & No conectado & MIs no conectados \\
\hline Nsaltos & \# saltos & Saltos \\
\hline Nsmax & Max \# de saltos & Max \# Saltos \\
\hline dmin & Min distancia & Distancia mínima \\
\hline$a, b$ & Variable $\mathrm{i}, \mathrm{j}$ & Variable temporal \\
\hline $\mathbb{U} j$ & subconjunto & Subconjunto de U \\
\hline
\end{tabular}
a un transformador de distribución.

\section{Tabla 2. Variables usadas para modelo OPDWHN-AMI}


En la tabla 3 se presenta un resumen de las variables empleadas en el modelo de planeación de redes eléctricas de distribución.

Tabla 3. Variables usadas en modelo DORED

\begin{tabular}{|c|c|c|}
\hline Símbolo & Descripción & Significado \\
\hline$C_{t r}$ & Costo & $\begin{array}{l}\text { Costo de transformador de } \\
\text { distribución }\end{array}$ \\
\hline$C_{\text {sub }}$ & Costo & Costo total de la subestación \\
\hline$C m v_{c o n}$ & Costo & Costo total del conductor MV \\
\hline$C b v_{c o n}$ & Costo & $\begin{array}{l}\text { Costo total del conductor de la } \\
\text { red de bajo voltaje }\end{array}$ \\
\hline$d$ & distancia & $\begin{array}{l}\text { Distancias máximas } \\
\text { requeridas en cada tramo }\end{array}$ \\
\hline$d \max$ & Distancia & Distancia máxima permitida \\
\hline$z e$ & Variable & $\begin{array}{l}\text { Número de elementos de cada } \\
\text { clúster }\end{array}$ \\
\hline$C_{p}$ & Demanda en kVA & $\begin{array}{l}\text { Demanda individual según su } \\
\text { estrato }\end{array}$ \\
\hline le & Variable & Número de clústeres \\
\hline$d_{a}$ & Distancia & $\begin{array}{l}\text { Longitud de un tramo de un } \\
\text { punto } i \text { a un punto } j\end{array}$ \\
\hline$N$ & Variable & $\begin{array}{l}\text { Contiene el valor de la } \\
\text { longitud de del vector de } \\
\text { Usuarios y transformadores }\end{array}$ \\
\hline$k$ & Variable costo & $\begin{array}{l}\text { Costo por cada metro } \\
\text { instalado en red MV }\end{array}$ \\
\hline$\phi_{i}$ & Subconjuntos & Subconjuntos de agrupaciones \\
\hline$\tau$ & Elementos de un clúster & Max \# permitido en un clúster \\
\hline$\beta_{i}, \beta_{j}$ & Coordenadas & $\begin{array}{l}\text { Coordenadas en latitud y } \\
\text { longitud de usuarios }\end{array}$ \\
\hline$A(\beta)$ & Conjunto & $\begin{array}{l}\text { Usuarios emplazados en área } \\
\text { georreferenciada }\end{array}$ \\
\hline$\Omega$ & Variable & $\begin{array}{l}\text { Número de elementos a } \\
\text { desplegarse }\end{array}$ \\
\hline$\mu$ & variable & Longitud de la zanja \\
\hline$\pi$ & conjunto & $\begin{array}{l}\text { Número de pozos a } \\
\text { desplegarse }\end{array}$ \\
\hline
\end{tabular}

En la Figura 3 se presenta el flujograma del algoritmo Despliegue Óptimo de Redes Eléctricas de Distribución (DORED), el cual, contiene la metodología del modelo propuesto en el presente documento. Se parte del análisis y extracción de los datos georreferenciados obtenidos de la plataforma virtual gratuita OpenStreetMap. Posteriormente, mediante el software Matlab, se procede a preparar el conjunto de datos a ser utilizados, es decir, no toda la información es válida, puesto 
que, en un archivo osm existen miles de coordenadas georreferenciadas (Latitud y longitud), de tal manera que, si no se hace una limpieza de datos, estos hacen que, el cpu-time que el ordenador emplee para encontrar la solución, aumente de manera significativa arrojando resultados poco confiables. Por lo tanto, es de mucha importancia ordenar la información y desechar datos que se encuentran fuera del área de interés para el despliegue óptimo de redes eléctricas de distribución.

Una vez que la información se encuentra clasificada se definen las variables a ser empleadas en el modelo. Estas variables son: coordenadas de viviendas, ubicación de subestaciones, sitios candidatos para emplazar los transformadores de distribución y datos de las vías disponibles para la construcción del mínimo árbol de expansión de la zanja, logrando generar con ello, la topología necesaria requerida en los primarios de los transformadores. En consecuencia, una vez definida las variables a emplear en el modelo, se genera una matriz de distancias de dimensión nxn (matriz cuadrada) que arma un mesh relacionando a todos los nodos entre sí. Para el cálculo de las distancias se emplea la ecuación de haversine, anteriormente mencionada, en la ecuación 1.

Una vez que se conoce la matriz de distancias se procede a generar varios escenarios posibles, es decir, se generan subconjuntos de soluciones previas. Además, estas soluciones previas se apoyan en técnicas de clusterización, específicamente en una variante del algoritmo de PRIM, el cual, construye árboles de mínima expansión. El aporte en la variante del algoritmo de PRIM, antes mencionada, radica en la integración de rutinas de verificación, el cual, permite ingresar restricciones de capacidad y rangos máximos permitidos de cobertura. Por lo tanto, mediante la variante de PRIM el modelo es capaz de agrupar nodos (usuarios finales, transformadores, subestaciones, sitios candidatos., etc.) y generar escenarios previos. El número de escenarios previos generados dependerá el número de sitios candidatos para emplazar transformadores de distribución, estos sitios candidatos son los pozos distribuidos a lo largo de la zanja, por la cual, pasará la red de medio voltaje. 


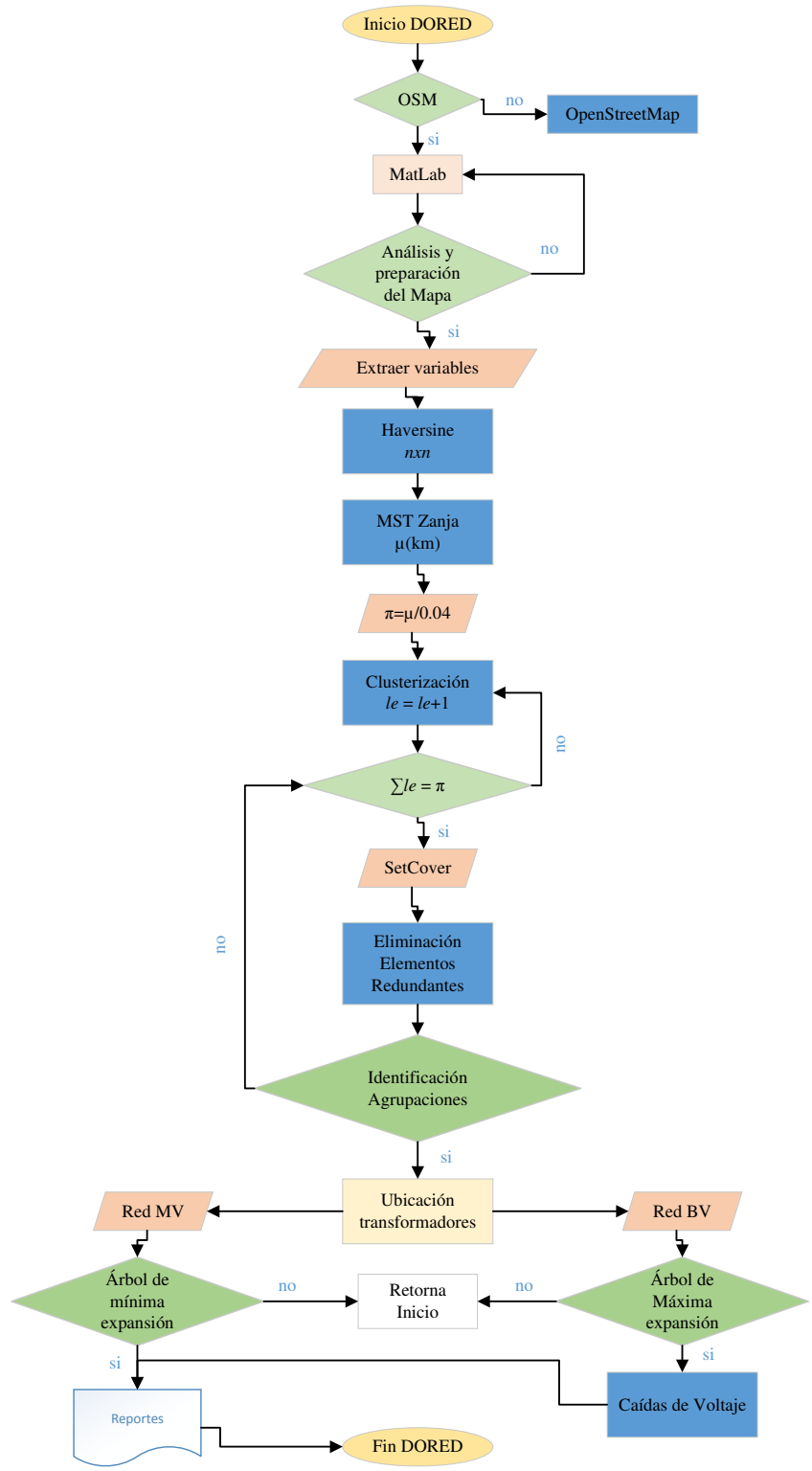

Figura 3. Flujograma del algoritmo dimensionamiento óptimo de redes eléctricas de distribución (DORED) 
Un detalle de mucho interés, es que, la zanja podrá ser usada para el despliegue de fibra óptica. La distribución de los pozos se da a 40 metros y su emplazamiento se origina desde la subestación hacia toda la ruta que sigue la zanja en el caso de redes eléctricas soterradas. A manera de ilustración, si la zanja dispone de $5 \mathrm{~km}$ lineales desde la subestación hacia todos sus ramales el número de pozos requerido mínimo en el despliegue será de 125 excavaciones. Por lo tanto, los escenarios estarán representados por una matriz de $m n x$ elementos, es decir, sus filas y columnas son de diferente longitud, donde, $n$ representa a la cantidad de pozos y $m$ el número de usuarios finales emplazados en el área de interés.

Una vez identificados los diferentes escenarios previos generados, se procede a ingresar dicha matriz al algoritmo de setcover, el cual, minimiza el número de escenarios generados garantizando la máxima cobertura a los usuarios finales. Es decir, se toman los subconjuntos que contengan el mayor número de nodos cubiertos hasta que todos los usuarios desplegados en el escenario queden asociados a algún subconjunto. En este punto se genera un inconveniente, recordemos que los escenarios son generados mediante una técnica de clusterización observando los mismos criterios de capacidad y cobertura en cada nueva iteración, lo que hace que, existan subconjuntos seleccionados como solución por el algoritmo del setcover que contengan los mismos elementos que otros subconjuntos, es decir, en la solución del algoritmo del setcover tendremos subconjuntos con elementos redundantes, lo que, en redes de distribución desemboca en la posibilidad de que un usuario pueda estar cubierto por más de 1 transformador de distribución, lo cual, en la práctica no es correcto.

Por lo tanto, una vez identificado los conjuntos se procede a eliminar los elementos redundantes siguiendo la metodología como se detalla a continuación; se ordenan los subconjuntos de mayor a menor y en cada iteración verificamos que el subconjunto siguiente no contengan los elementos del subconjunto predecesor de la matriz solución obtenida por el algoritmo Setcover. Este mismo criterio se aplica hasta hacer un barrido en toda la matriz, logrando con ello, eliminar los elementos redundantes garantizando que la capacidad de albergar usuarios sea la máxima posible en cada subconjunto. 
Por lo tanto, como resultado tendremos el mínimo número de subconjuntos con la máxima cobertura posible a cada usuario final. Si un usuario se queda sin conexión a algún secundario de los transformadores a emplazarse, el diseñador conocerá toda la información necesaria, de tal manera que, pueda tomar decisiones adecuadas para otorgarle conectividad a algún secundario de los transformadores de distribución distribuidos en el área de interés. Una vez identificados los subconjuntos, con sus elementos redundantes eliminados, se procede a emplazar los transformadores de distribución. En consecuencia, el número de transformadores requeridos en el despliegue corresponde a la cantidad de subconjuntos resultantes del algoritmo del setcover.

Dicho de otra manera, tomando el mismo ejemplo anterior, en el que, se necesitaba 125 pozos para una longitud de $5 \mathrm{~km}$ de zanja, el algoritmo setcover arrojará como resultados posiblemente 80, 75, 50 (subconjuntos) unidades de transformadores requeridos en el escenario, esta variación dependerá exclusivamente de las restricciones de capacidad y cobertura ingresadas como restricciones en la generación inicial de los subconjuntos previos, antes de pasar por el algoritmo del setcover.

En consecuencia, si aumentamos la capacidad de agrupar elementos disminuiremos la cantidad de transformadores de distribución requeridos, pero, indudablemente aumentará la capacidad en kVAs requerida a instalar en dicho punto, caso contrario si, disminuimos la capacidad de agrupar aumenta el número de transformadores necesarios en el despliegue y disminuye la capacidad en kVAs de los transformadores necesarios en el despliegue.

Por lo tanto, se logra optimizar el número de transformadores mediante una técnica de clusterización, con la cual, se genera escenarios y posteriormente, mediante el algoritmo del setcover reducimos al máximo los subconjuntos necesarios para garantizar la máxima cobertura a los usuarios finales y, posteriormente se procede a ubicar los transformadores, en cada subconjunto, aproximadamente en el centro de masa, con la finalidad de garantizar distancias similares desde el transformador hacia sus puntos de carga más alejados.

En este punto, el que se ha logrado tener un conocimiento sobre de la distribución de los elementos de potencia en redes eléc- 
tricas de distribución, se procede a generar árboles de mínima y máxima expansión a dos capas. Una capa en medio voltaje (mínima expansión) y la otra capa en bajo voltaje (máxima expansión), de tal manera que, la red de distribución óptima queda identificada. Por lo tanto, hasta este punto se logra proporcionar mediante el software Matlab y con la base de datos de OpenStreetMap un mapa de ruta, donde, mediante restricciones, tales como capacidad y cobertura, se garantiza que su costo sea el mínimo.

Una vez que se conoce la topología de la red eléctrica de distribución y cada uno de sus subconjuntos se procede a verificar el comportamiento de la caída de tensión desde el transformador hacia los nodos de carga más lejanos en cada subconjunto. Para ello, se ha otorgado cargas randómicas normalizadas a cada usuario. Estas cargas proporcionadas aleatoriamente corresponden a valores tipo considerando la introducción de cocinas de inducción y calentamiento de agua. Además, se ha dividido el área en sectores, es decir, se proporcionará valores randómicos de cargas tipo identificando los estratos a los que pertenece cada usuario, los valores de consumo en kVAs de cada estrato, se tomó de la Guía de diseño de la EEQ. Por lo tanto, el modelo es capaz de hacer el análisis de caídas de tensión desde los secundarios del transformador hacia sus puntos de carga más alejados y, a su vez, puede determinar la capacidad del transformador con valores ya estandarizados. El modelo nos permitirá ver y analizar el comportamiento, si los usuarios aumentan su demanda, ayudando con eso a planear posibles expansiones por incremento de energía demandada, que con el pasar del tiempo, su tendencia está en aumento. Finalmente, el modelo entrega un reporte ejecutivo de los elementos requeridos para la construcción de la red eléctrica de distribución.

\section{Análisis de resultados}

\section{A. Red heterogénea inalámbrica para medición inteligente}

en esta sección, se demuestra como el modelo de planeación de una red heterogénea inalámbrica que despliega PADs y los MIs que finalmente se articular hasta lograr la conectividad con una estación base celular. Se evalúan parámetros que dan cuenta del 
rendimiento en términos de minimización de los recursos. La Figura 4 muestra el despliegue de los PADs candidatos, los mismos que se ubican en las intersecciones de calles y avenidas, lugar donde se ubican postes de alumbrado público y donde finalmente se podrán instalar los PADs requeridos para dar cobertura a los MIs de cada una de las viviendas, considerando la capacidad de los PADs en función de la tecnología inalámbrica en cada MI o PAD.

El escenario advierte un número inicial de $\mathrm{PADs}$ con $=57$, un número de MIs $=349$ y estación base celular $=1$. El escenario se ha dividido por un proceso de teselación con Delaunay y Voronoi para segmentar las áreas que pueden determinar los estratos o grupo de viviendas, según detalla la Figura 5

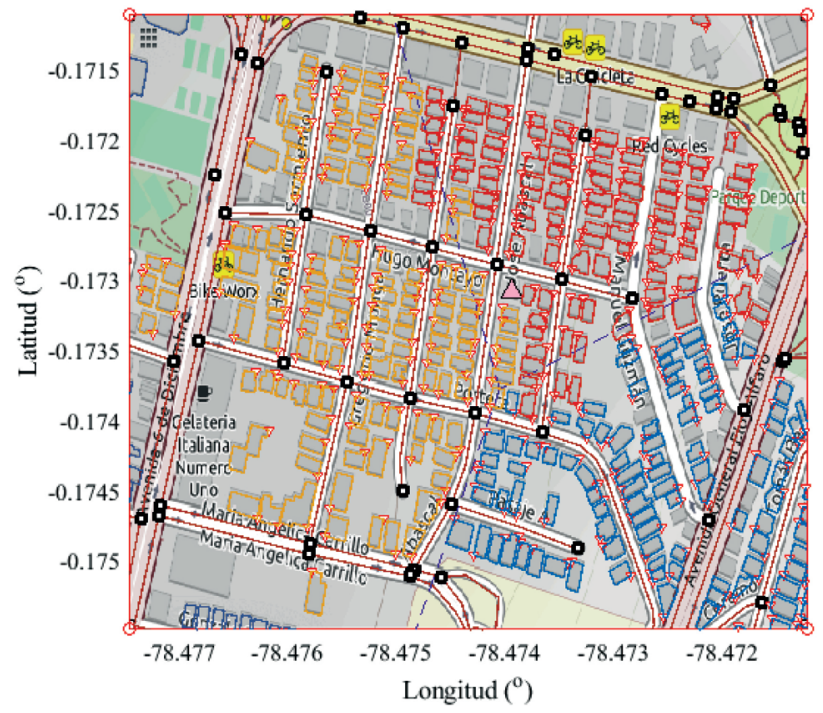

$\nabla$ MIs o PADs Candidatos $\triangle$ EB --- Estratos

Figura 4. Escenario de planeación de una red heterogénea inalámbrica

Además, la Figura 5 indica la reducción del número $M$ de PADs que se requieren para cubrir un número $N$ de MIs; se aprecia también el proceso de teselación realizada con Delaunay y Voronoi para dividir en 3 áreas o estratos que dividen los diferentes tipos de clientes que puede segmentar una empresa de distribución eléctrica; en este caso, cada estrato toma un color diferente. 


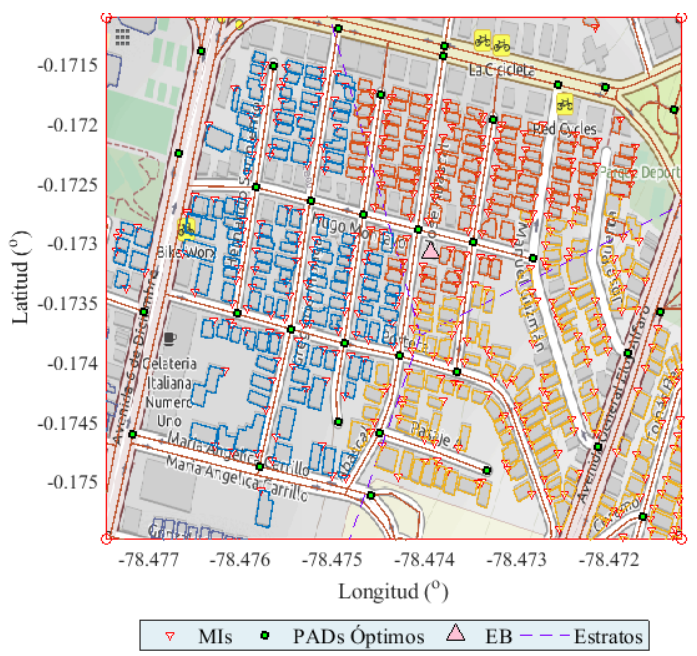

Figura 5. Optimización de recursos - minimización de PADs

La Figura 6 representa la malla factible lograda para el escenario propuesto con una $d \mathrm{~min}=49 \mathrm{mts}$; de esta manera, se consigue la conectividad del grafo $G=(V, E)$. Con esta visión previa de los posibles enlaces inalámbricos se procede a buscar el mínimo árbol de expansión que se podría lograr.

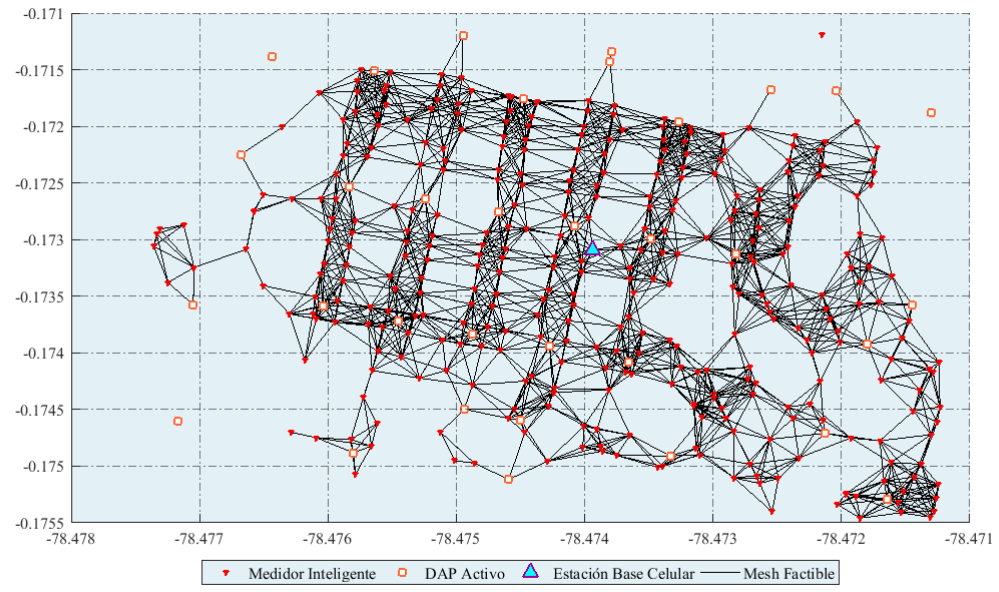

Figura 6. Malla factible de conexiones posibles con restricción de distancia 
La Figura 7 muestra la reordenación inversa Cuthill-McKee para mover todos los elementos distintos de cero más cerca de la diagonal, reduciendo el "ancho de banda" de la matriz original G.

La Figura 8 muestra el mínimo árbol de expansión, luego de haber resuelto el problema del setcover que logra la mayor cobertura; es decir, maximiza la cobertura de todos los MIs con la mínima solución encontrada al generar el mínimo árbol de expansión a través del algoritmo $\Psi$ para generación del árbol.

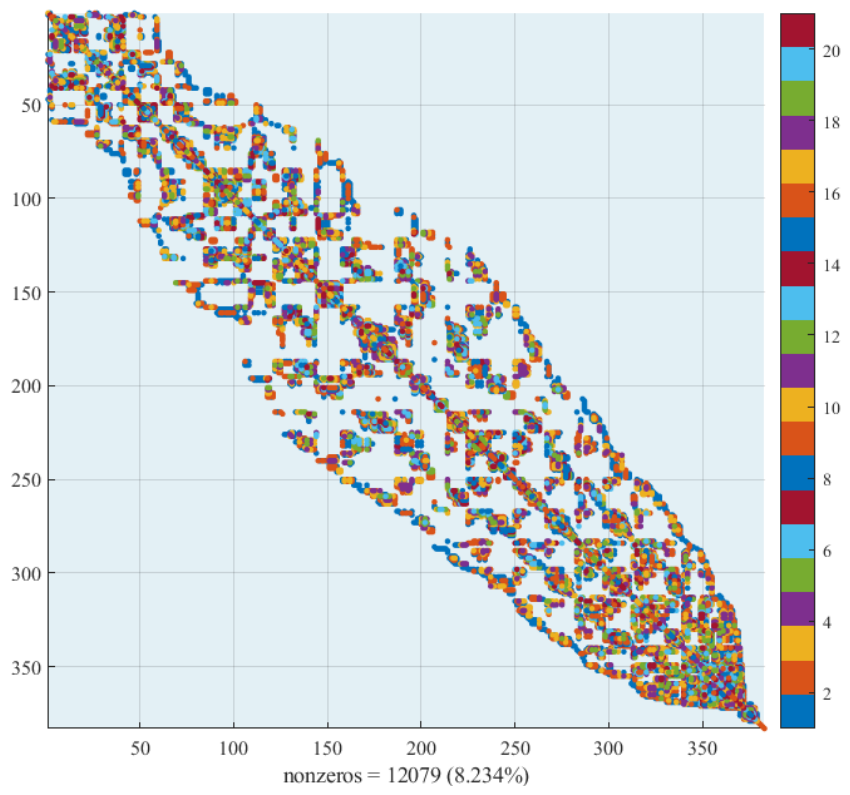

Figura 7. Reordenación inversa Cuthill-McKee

La Figura 9 representa los subconjuntos obtenidos al haber incluido tres tipos de tecnologías inalámbricas y en conjunto se puede visualizar los recursos que son agrupados y que a través de un PAD finalmente llegan hasta una estación base celular, la representación de este proceso se encuentra en color celeste. Los enlaces de tecnología inalámbrica WiFi se encuentran en color rosado; los enlaces entre PAD en color verde, para este caso la tecnología inalámbrica puede ser WiFi o Celular dependiente de las características de la zona y los recursos existentes en términos de cobertura celular. 


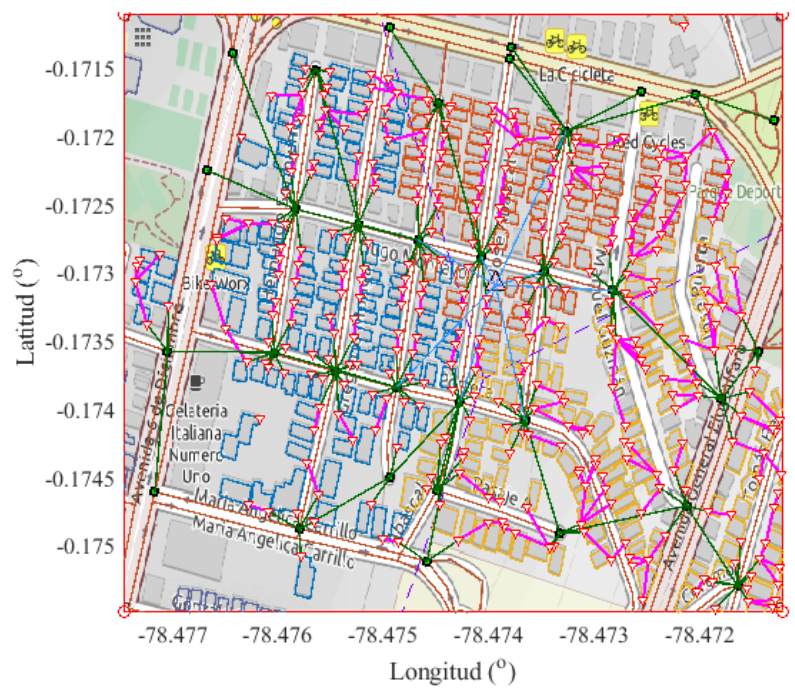

$\nabla$ MIs $\bullet$ PADs Óptimos $\triangle \mathrm{EB}-$ Enlace Inalámbrico

\section{Figura 8. Red Despliegue de la Red Heterogénea Inalámbrica}

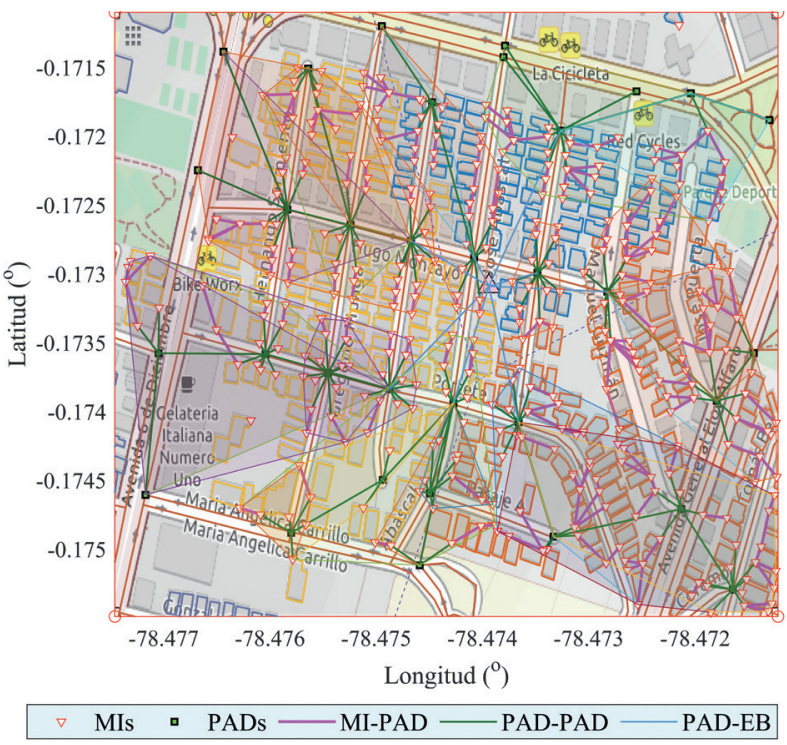

Figura 9. Subconjuntos de MIs por cada PAD 


\section{B. Red de Distribución Eléctrica Soterrada}

En la Figura 10 se presenta la disposición del escenario, el cual, se empleará para desplegar transformadores eléctricos de distribución. El escenario propuesto dispone de 3 estratos, ilustrados en la Figura 10 mediante tres colores diferentes. El criterio de estratificación se lo llevó a cabo mediante el algoritmo de K-medoids tomando en consideración la guía de diseño de redes de distribución de la Empresa Eléctrica Quito (EEQ) para otorgar demandas máximas tipo por cada usuario. Los estratos empleados en el presente documento son: E, D, $\mathrm{C}$ y B. Es de mucha importancia mencionar que, el modelo, permite modificar dichos parámetros para poder ajustarse a diferentes realidades o disposiciones topográficas. A cada usuario de cada estrato se le otorgó un consumo referente máximo randómico normalizado.

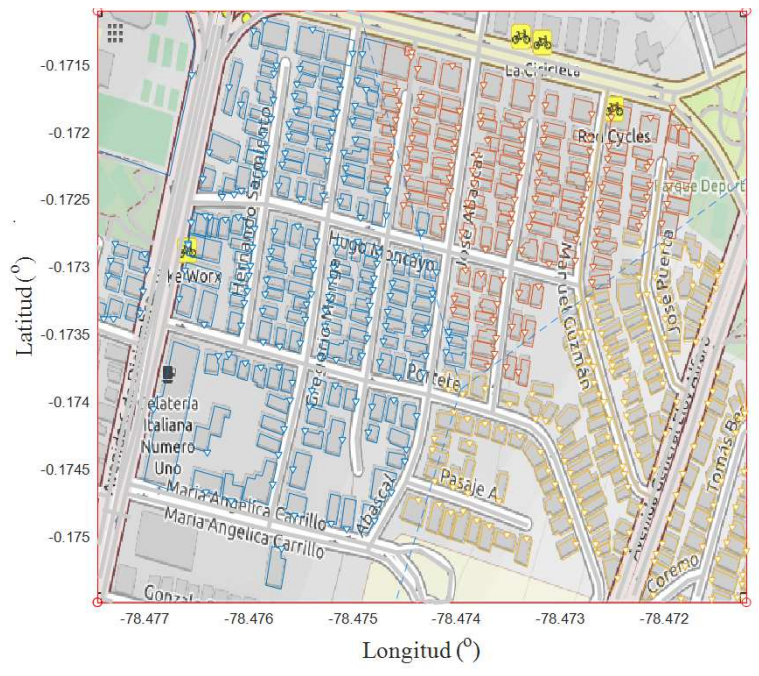

Figura 10. Escenario inicial considerando estratificación

En la tabla 4 se ilustra un cuadro resumen de los resultados obtenidos del despliegue óptimo de transformadores para satisfacer de servicio eléctrico a los abonados al sistema de distribución, considerando, diferentes escenarios. La heurística propuesta, para resolver el problema combinatorio de ubicación de transformadores observando restricciones de capacidad y cobertura, se valida mediante la tabla 
4, donde, se puede observar los diferentes escenarios resueltos por el modelo. Se puede apreciar además que, a medida que, la capacidad aumenta los parámetros de las columnas 2, 4 y 5 también lo hacen. En el caso de la columna 2 de la tabla 4 se desvela que, al incrementar la capacidad la variación de la cobertura no sigue ningún patrón, es decir, no es ni directamente proporcional ni inversamente proporcional a la capacidad, por lo tanto, el porcentaje de cobertura no depende de la capacidad de agrupamiento del transformador, sino que, depende de la disposición geográfica de los usuarios finales, puesto que, la ubicación involucra directamente a la variable distancia, que es, una restricción tonada en cuenta en el presente modelo.

Tabla 4. Planeación y despliegues obtenidos variando capacidad de agrupamiento de cada transformador

\begin{tabular}{|c|c|c|c|c|}
\hline $\begin{array}{l}\text { Capacidad } \\
\text { Máxima } \\
\text { (unidades) } \\
\end{array}$ & $\begin{array}{c}\text { Cobertura } \\
(\%)\end{array}$ & $\begin{array}{c}\text { Cap. Subestación } \\
\text { (MVAs) }\end{array}$ & $\begin{array}{c}\text { Transformadores } \\
\text { Requeridos } \\
(\mathrm{kVA} / \text { cantidad }) \\
\end{array}$ & $\begin{array}{l}\text { kVA-m } \\
\text { máximo }\end{array}$ \\
\hline 10 & 83.77 & 1.42 & $\begin{array}{c}45 / 19 \\
60 / 5 \\
15 / 7 \\
50 / 5 \\
30 / 5\end{array}$ & $\begin{array}{l}493.17 \\
325.43 \\
103.34 \\
400.12 \\
476.17\end{array}$ \\
\hline 15 & 82.56 & 1.44 & $\begin{array}{c}100 / 7 \\
75 / 5 \\
60 / 7 \\
45 / 2 \\
30 / 2 \\
50 / 1 \\
15 / 4\end{array}$ & $\begin{array}{l}513.22 \\
448.40 \\
603.94 \\
242.16 \\
219.40 \\
212.47 \\
222.31\end{array}$ \\
\hline 18 & 90.79 & 1.42 & $\begin{array}{c}100 / 9 \\
75 / 6 \\
60 / 1 \\
45 / 2 \\
30 / 2 \\
15 / 7 \\
112.5 / 2\end{array}$ & $\begin{array}{l}636.45 \\
592.41 \\
694.19 \\
458.53 \\
222.26 \\
171.51 \\
596.00\end{array}$ \\
\hline
\end{tabular}


40

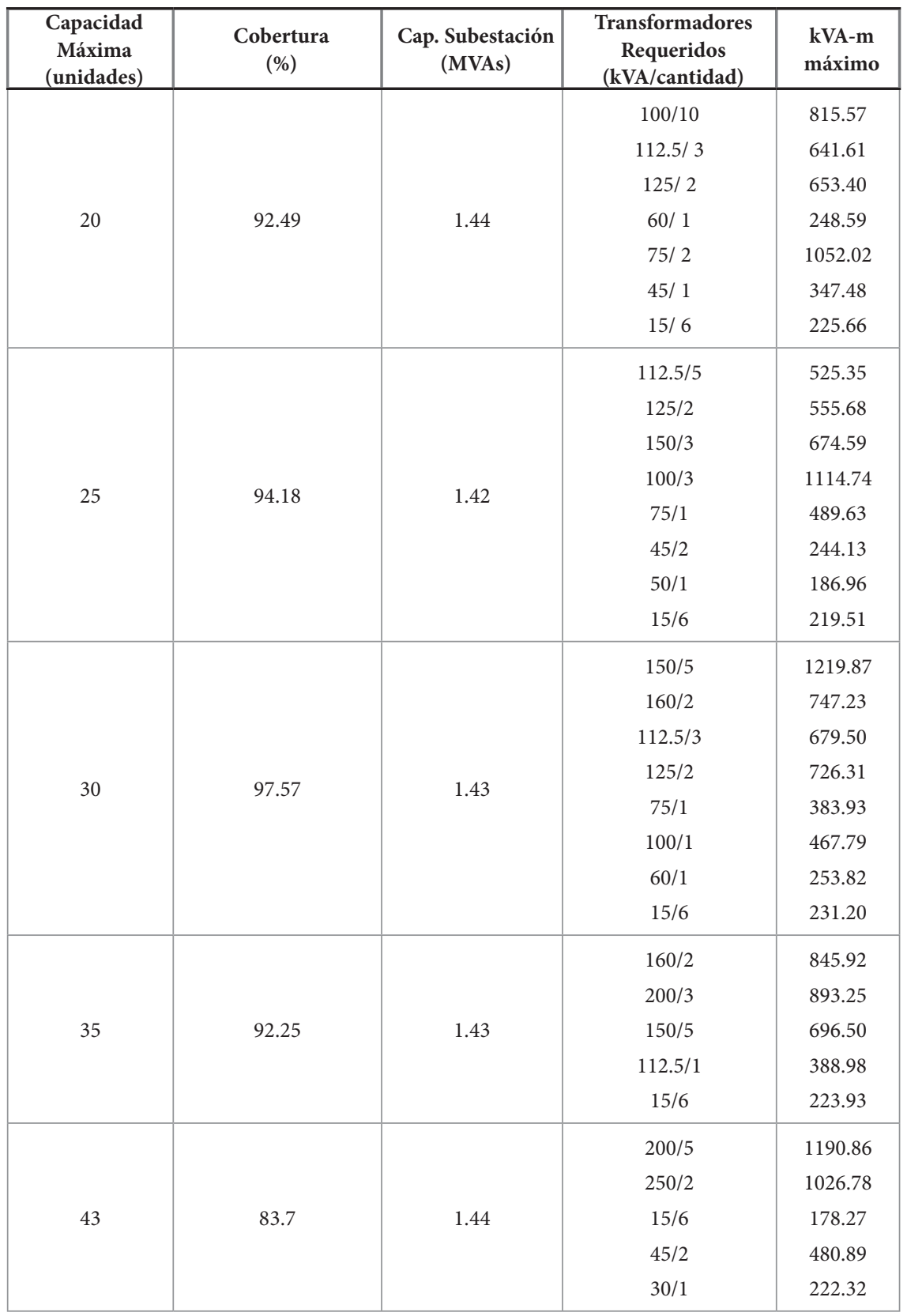


En la columna 3, de la tabla 4, se presenta la capacidad calculada por el algoritmo de la subestación requerida en MVAs. En párrafos anteriores se ha explicado que, a cada usuario, se otorgó un consumo máximo tipo randómico, lo que hace que, en cada iteración la heurística obtenga diferentes resultados muy próximos entre sí. Dichos resultados son controlados, es decir, se otorga por cada estrado un nivel mínimo y máximo de consumo randómico a cada usuario. Por lo tanto, la heurística propuesta no coloca cargas rígidas o únicas a los usuarios finales y además permite identificar, por la posición geográfica, el estrato al que pertenece cada abonado a la red eléctrica de distribución. En consecuencia, la capacidad de la subestación no depende de la capacidad en MVAs estandarizado de cada transformador, sino que, depende de la carga total instalada y del número y tipo de usuarios. Por lo tanto, el valor obtenido de la tabla 4 en la columna 3 , debe ser estandarizado de acuerdo a la disposición del mercado en MVAs de subestaciones de distribución.

En la columna 4 de la tabla 4 se aprecia que, a medida que la capacidad aumenta, disminuye la cantidad de transformadores y aumenta la necesidad de incrementar la capacidad en MVAs requerida para satisfacer de servicio a usuarios finales asociados a la red eléctrica de distribución. Por lo tanto, queda demostrada la funcionalidad y validez de la heurística propuesta. En la columna 5 se presenta la métrica en kVA-m máximo desde un transformador hasta su punto más lejano, es decir, se presenta el peor de los casos. Mediante la métrica, expuesta anteriormente, se puede dimensionar el calibre del conductor a emplearse, considerando la caída de voltaje en los extremos más alejados desde la fuente (transformador de servicio), en bajo voltaje, mediante la relación kVAs-m calculados/kVAs-m soportados. 


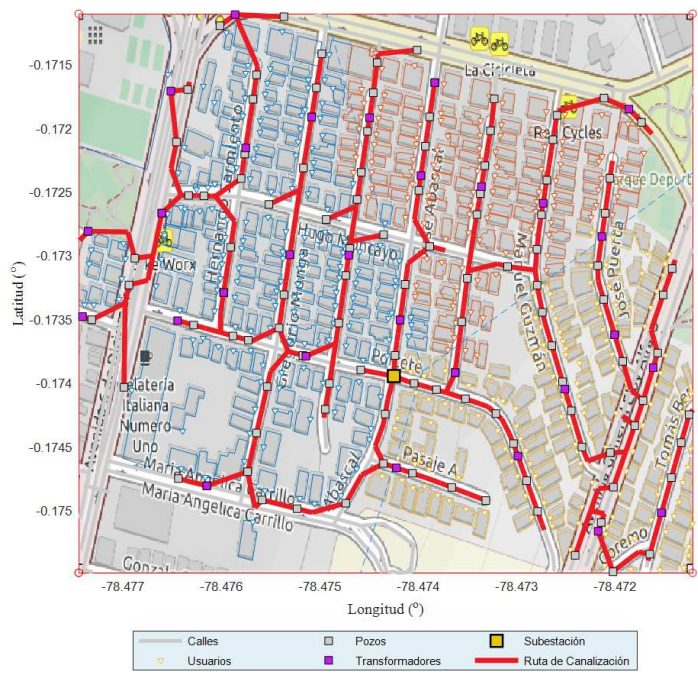

Figura 11. Planeación óptima de una red de distribución soterrada de energía eléctrica considerando capacidad y cobertura

En la Figura 11 se presenta el resultado de la planeación obtenida por la heurística propuesta en el presente documento. Mediante la figura, anteriormente mencionada, se puede apreciar el mapa de ruta a emplearse garantizando el mínimo costo. El escenario de la Figura 11. dispone de $\mathrm{N}=349$ usuarios distribuidos geográficamente y corresponde a los resultados obtenidos en la tabla 4 con capacidad máxima de albergar a 18 usuarios. Además, la heurística planteada proporciona datos de variables que son determinantes en el momento de elegir sobre la solución a ejecutarse en el proceso de planeación, tales como: número de pozos requerido (115), longitud de canalización o zanja $(4.614 \mathrm{~km})$ y demanda total máxima de cada estrato: 1 (431.44 kVA), 2 (371.22 kVA) y 3 (626.04 kVAs). En consecuencia, el modelo propuesto es capaz de modelar de manera dinámica el despliegue de transformadores eléctricos dentro de un área georreferenciada proporcionándonos parámetros valiosos de diseño con los cuales, el ingeniero eléctrico, puede tomar decisiones observando parámetros técnicos. Por lo tanto, en el presente documento se desvela una herramienta muy prometedora de diseño en 
redes eléctricas de distribución soterrada que ayude al diseñador a tomar decisiones, no solo más certeras, sino que, garantice la continuidad y calidad del servicio eléctrico a los usuarios finales pertenecientes a la red eléctrica de distribución.

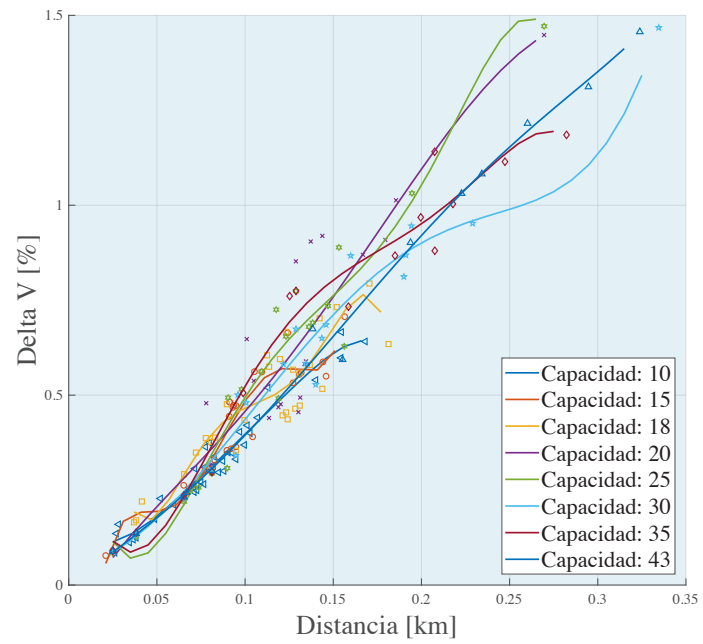

Figura 12. Máximas caídas de voltaje en la red secundaria en diferentes escenarios considerando capacidad y rangos de cobertura

En la Figura 12 se aprecia el comportamiento del voltaje en función de la distancia en la red de medio voltaje. Es evidente que la variación de voltaje, expresada en porcentaje, aumenta a medida que la distancia también lo hace, esto se debe a que, a mayor longitud del conductor existe mayores pérdidas por efecto Joule. Los datos de la Figura 12, en el escenario ensayado, están distribuidos de manera dispersa, puesto que, como se explicó en párrafos anteriores, los datos obtenidos no siguen ningún patrón a medida que incrementa la capacidad, sino que, depende del escenario, la capacidad de agrupamiento observando las restricciones propias del modelo propuesto y la capacidad de conducción del conductor a emplear. Además, la variación de voltaje no solo depende de la distancia, sino que, también depende de las distancias acumuladas a lo largo del tramo hasta llegar al usuario más alejado del circuito, del lado secundario del transformador al que pertenece. En consecuencia, se 
puede apreciar que a medida que se aumenta la capacidad los porcentajes de variación de voltaje también lo hacen y esto sucede, ya que, al aumentar la capacidad de albergar usuarios por un transformador de manera conjunta aumenta las distancias desde un transformador hacia su nodo más lejano de servicio. Por último, mediante la Figura 12, se aprecia que, a cada transformador en sus diferentes escenarios con sus respectivas variaciones de voltaje generales, de lo que, se puede inferir que ningún transformador supera los límites máximos de caídas de voltaje desde la fuente hacia el usuario más alejado del transformador en cada escenario propuesto.

En la Figura 13 se ilustra el modelado de la subestación empleada en el proceso de simulación de la red eléctrica en los primarios de los transformadores otorgándole criterios de confiabilidad, lo cual, naturalmente eleva los costos de implementación. En el modelado del mapa de ruta obtenido por la heurística mediante el software MATLAB, se ha extraído la información necesaria como: cargas concentradas en cada transformador, número y capacidades requeridas en MVAs de los transformadores para satisfacer a la demanda en cada tramo, dimensionamiento de la subestación requerida, número de alimentadores y longitudes en cada tramo, ya sea, en medio y bajo voltaje. En consecuencia, una vez obtenidos las variables necesarias para el diseño de despliegue óptimo de transformadores considerando las demandas totales tipo por cada abonado, mediante el software Cymdist se procede a simular un flujo de potencia. Mediante la simulación del flujo de potencia podemos verificar el estado de la red eléctrica en medio voltaje con las condiciones proporcionadas en el mapa de ruta obtenido de la heurística propuesta. Por lo tanto, en la Figura 13. se verifica, además de la configuración de la subestación, el flujo de potencia, logrando con ello, obtener los datos de las variables de voltajes en p.u. con sus respectivos ángulos, corrientes en los conductores, potencia activa y potencia reactiva, con lo que, se puede ejecutar procesos de planeación en redes eléctricas de distribución. Es muy importante mencionar que, los valores en p.u. son adimensionales y pueden representar a cualquier magnitud física, ya sea, voltaje, corriente, impedancias etc., en consecuencia, cada valor en p.u. adimensional depende de su valor base real de cualquier parámetro eléctrico al que nos refiramos, para convertir un valor en p.u., en valores reales. 


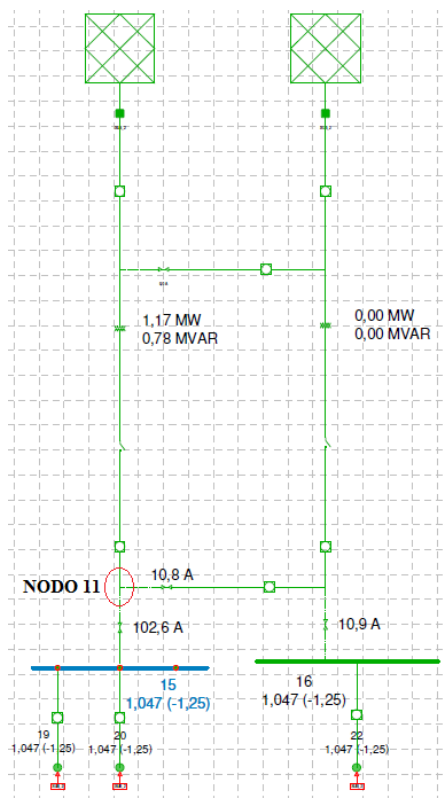

Figura 13. Modelado de la subestación mediante software cymdist

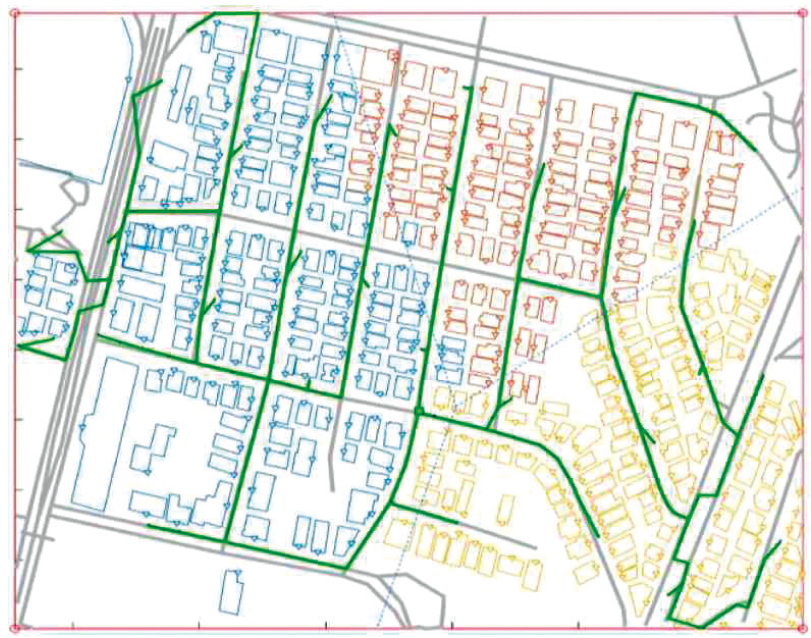

Figura 14. Diagrama unifilar trifásico en media tensión mediante software cymdist 
Mediante la tabla 4 y la Figura 13. se valida el funcionamiento de la heurística propuesta en el presente trabajo, puesto que, mediante el triángulo de potencias, con los datos de la Figura 13, nos permite encontrar, en MVAs, la capacidad de la subestación requerida para satisfacer la demanda bajo las consideraciones descritas anteriormente. En consecuencia, con los datos de potencia activa y reactiva obtenidos de la simulación en el software Cymdist, calculamos el valor de la potencia aparente, cuyo valor es 1.41 MVAs, mientras que, si nos percatamos en la tabla 4 en la fila con capacidad de 18 usuarios, la capacidad calculada por el modelo en MVAs es de 1.42. Por lo tanto, los valores obtenidos en el software Cymdist y los calculados mediante el modelo propuesto en el software MATLAB son semejantes, de tal manera que, se logra demostrar y validar la funcionalidad del modelo propuesto garantizando altos niveles de confiabilidad en los resultados obtenidos.

En la Figura 14 se muestra el diagrama unifilar obtenido en la simulación del flujo de potencia mediante el software Cymdist. En consecuencia, el mapa de ruta georreferenciado obtenido del software Matlab es posible simular, dicho mapa de ruta, en el software Cymdist con la finalidad de verificar el comportamiento de la red eléctrica en régimen permanente. Por lo tanto, una vez obtenido el mapa de ruta, mediante el software Cymdist, se puede realizar todo tipo de análisis pertinente en un proceso de planeación de redes de distribución, ya sea, soterrado, aéreo o mixto. Por lo tanto, la importancia de la propuesta planteada como una solución para despliegue de redes de distribución del presente documento, radica en que, mediante la observación de restricciones emplace los transformadores de potencia en los sitios idóneos garantizando minimizar costos maximizando la calidad y seguridad energética proporcionada a los usuarios finales. 


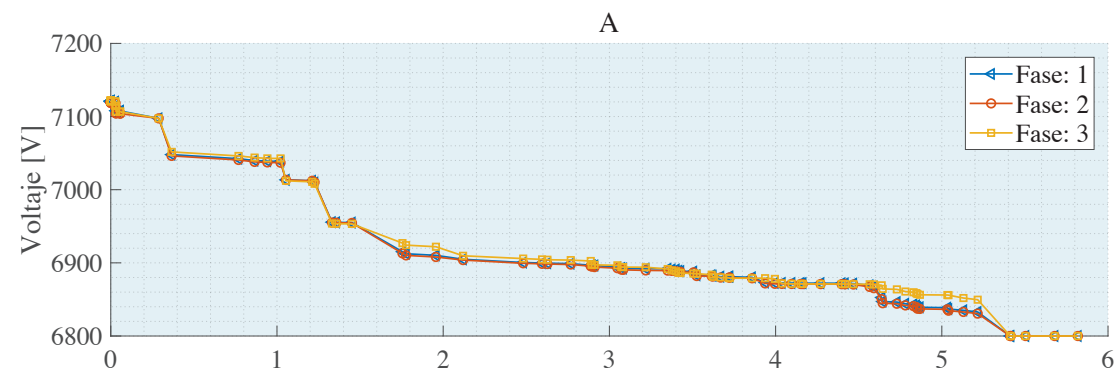

B

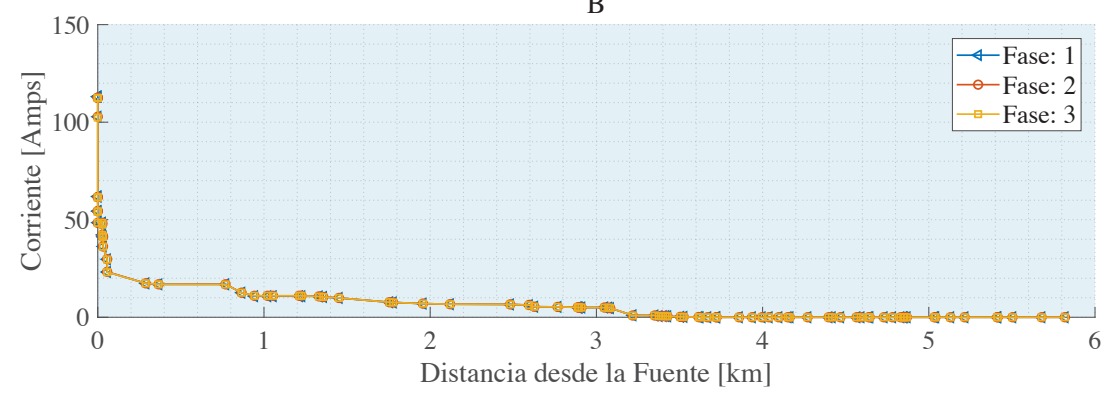

Figura 15. Perfil de voltaje y corriente circulantes en cada tramo desde la fuente

En las métricas de la Figura 15 se puede apreciar el comportamiento del voltaje y la corriente en función de la distancia. La longitud máxima desde la fuente hacia el transformador más alejado es aproximadamente $580 \mathrm{~m}$. En la Figura 15A se expresa que a medida que un transformador se aleja de la fuente existe caídas de voltaje en cada tramo, además, las caídas de voltaje en cada son similares, lo que pone de manifiesto que, para la presente explicación se ha modelado un sistema trifásico con cargas repartidas equilibradas en cada fase. Por otro lado, la Figura 15B nos muestra las máximas corrientes soportadas en la fuente y a medida que la distancia aumenta esas corrientes van disminuyendo, esto sucede ya que, en la fuente se refleja el consumo total de la zona a la que, dicha fuente, sirve. Por lo tanto, a medida que la distancia de servicio aumenta la corriente disminuye, ya que, la corriente nominal desde la fuente, en cada nodo primario, dependiendo de la topología se va dividiendo. 
En la Figura 16 A y B sucede algo similar que lo explicado en el párrafo anterior. En consecuencia, a medida que la distancia aumenta, tanto la potencia aparente como la potencia reactiva, disminuyen. Estas métricas nos permiten observar el estado de la red eléctrica de distribución y planificar la colocación de bancos de condensadores en caso de ser necesario.

A

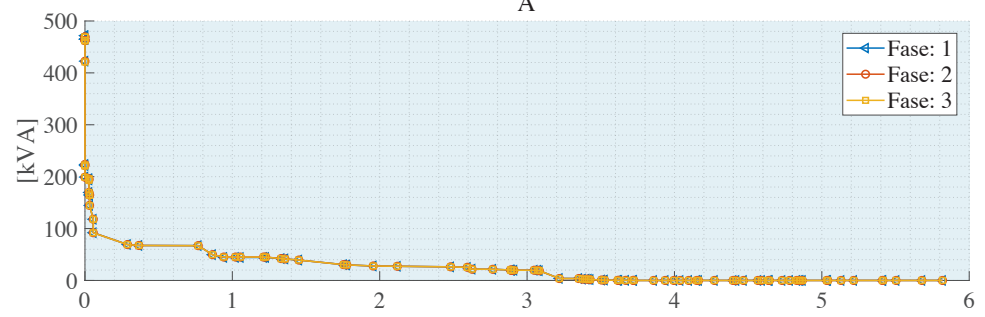

B

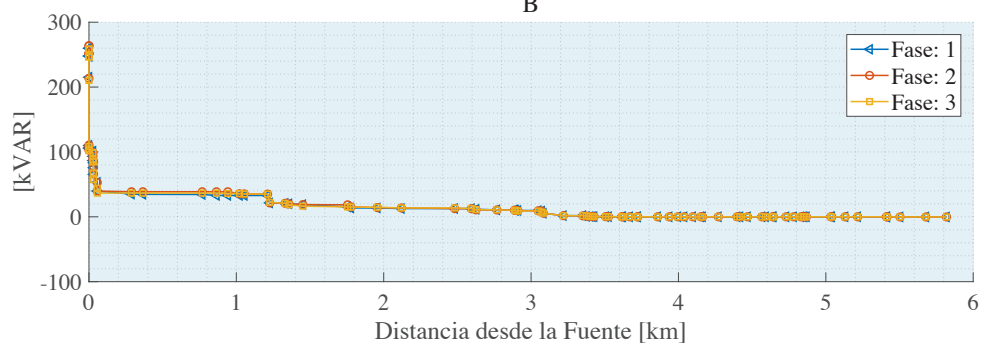

Figura 16. Perfiles de potencia aparente y reactiva desde la fuente en función de la distancia

En la Figura 17 se ilustra el diagrama unifilar al aplicar, mediante el software Cymdist, el módulo de análisis de fallas "Huecos de Tensión". Cada color representa la magnitud en p.u del hueco de tensión en cada tramo. En consecuencia, el color rojo simboliza mayor que 0 y menor que 0.1 , el color naranja mayor que $0.2 \mathrm{y}$ menor que 0.3 , el color verde mayor que 0.5 y menor que 0.6 y por último el color azul mayor que 0.9 y menor que 1 . 


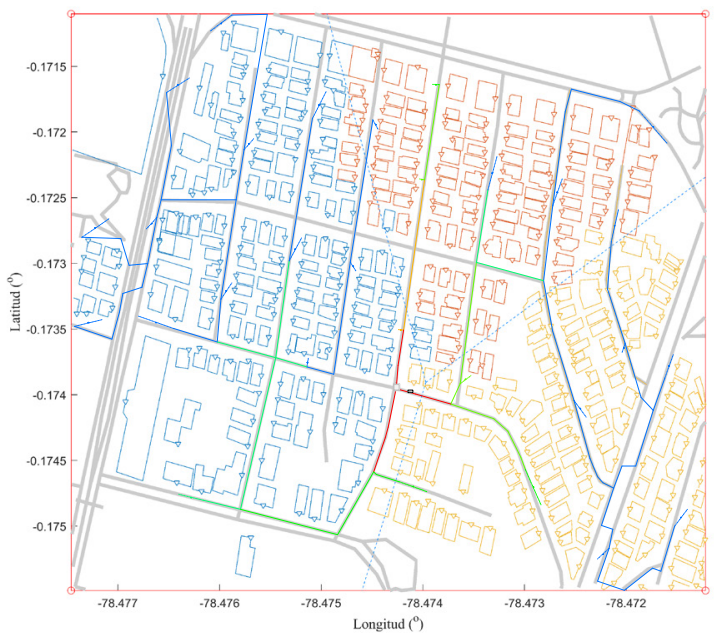

Figura 17. Análisis de huecos de tensión en la red de media tensión mediante software cymdist

Sabemos que, los huecos de tensión son perturbaciones en los sistemas eléctricos que afecta a la calidad del suministro de energía eléctrica. En consecuencia, el análisis de los huecos de tensión se produce en forma repentina en un punto particular de la red, para el caso, se ha hecho el análisis de hueco de tensión en el Nodo 11 (Ver Figura 13). Por lo tanto, los huecos de tensión se producen por la reducción súbita de la tensión recuperando sus valores habituales en un intervalo corto de tiempo y la causa habitual para que esto suceda son los cortocircuitos.

Por lo tanto, los huecos de tensión pueden influir en la impedancia y localización de falla, la forma de actuación de las protecciones, la conexión de la carga y el tipo de fallo. Además, provoca fallos en los equipos conectados a la red eléctrica, puesto que, existen dispositivos que necesitan cierta calidad de alimentación y pueden verse perjudicados en su rendimiento. 


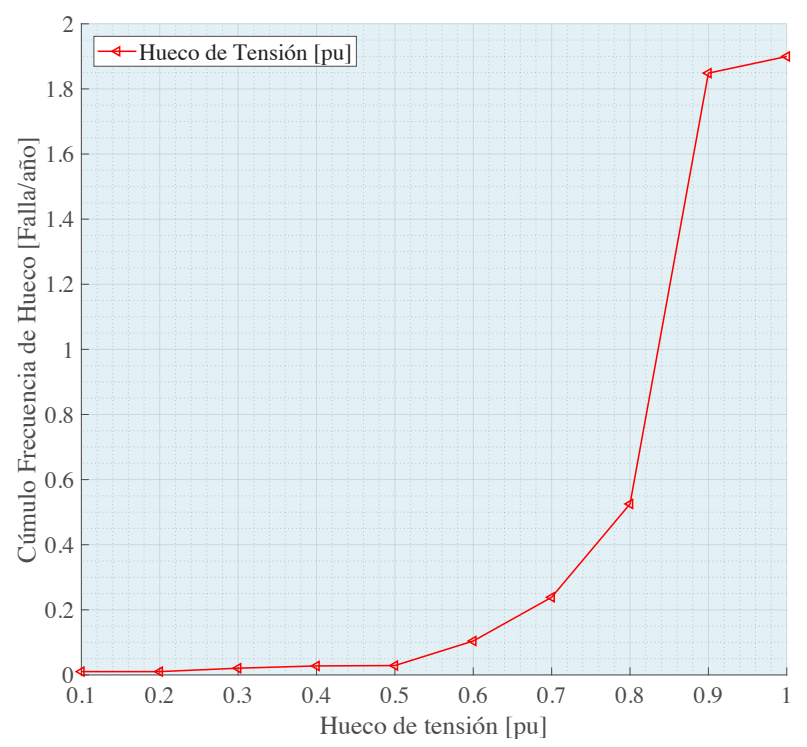

Figura 18. Suma de las frecuencias y su tensión de hueco

Como se ha explicado en párrafos anteriores un hueco de tensión está relacionado con la reducción del valor rms por un periodo corto de duración. Los valores relacionados en p.u. de la reducción de la componente fundamental de tensión son de 0.1 a 0.9 , con duración mayor que medio ciclo y menor de 1 minuto.

En la Figura 18 se ilustra en el eje de las ordenadas la suma de huecos de tensión esperados por año y en el eje de las abscisas las magnitudes de los diferentes huecos de tensión. En consecuencia, el cúmulo de frecuencia de huecos de tensión da cuenta, de cuántos huecos de tensión de valores remanentes menores o iguales a cierto umbral ocurrieron en un periodo de tiempo.

Es necesario mencionar que, el cúmulo de frecuencia al estar relacionado al número de fallas durante un año (tasa de falla) Cymdist modela este fenómeno en la red de distribución eléctrica con el ingreso de datos de las tasas de falla tanto de las barras como porciones de las líneas, cada posición de falla puede tener una tasa de falla diferente, la misma que, se puede relacionar directamente con el tipo de instalación. Las tasas de fallas son también diferenciadas 
en los tipos de falla de podrían producirse en los sistemas eléctricos de potencia, trifásico, monofásico, bifásico y bifásico a tierra.

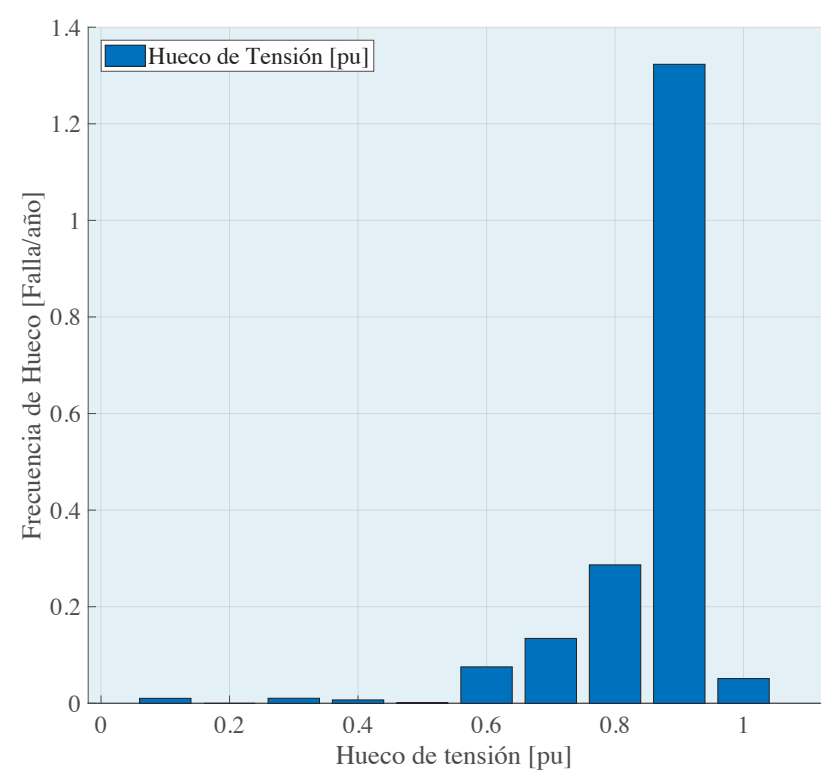

Figura 19. Número de fallas por año con respecto a la magnitud del hueco

De manera similar, en la Figura 19. se ilustra el número de fallas ocurridas al año con respecto a la magnitud del hueco de tensión. Es de mucha importancia citar que, el análisis de huecos de tensión simulado mediante el software Cymdist se aplicó en el Nodo 11, mismo que, se ilustra en la Figura 13.

Por lo tanto, queda demostrado que en el presente documento se logra, mediante el software Matlab, implementar una heurística capaz de dar solución óptima al problema de planeación de redes de distribución considerando restricciones de capacidad y cobertura otorgando confiabilidad a la solución obtenida, puesto que, se ha validado dicha solución mediante el software Cymdist. En consecuencia, la heurística propuesta es una herramienta de mucha utilidad para proporcionar soluciones optimas de planeamiento, utilizando teoría de grafos, en redes eléctricas de distribución. Además, mediante simuladores eléctricos, el diseñador, puede garantizar la viabilidad del proyecto minimizando los costos de implementación. 


\section{Conclusiones}

El modelo de planeación de redes heterogéneas inalámbricas conseguido permite visualizar un mapa de ruta para que empresas de distribución eléctrica avizoren un panorama mucho más claro en lo que respecta al despliegue de la infraestructura de medición avanzada que dará paso a medición inteligente de energía eléctrica.

El modelo propuesto es escalable y flexible, lo que, permite incorporar nuevos medidores inteligentes; incorporar tecnologías inalámbricas según sea la necesidad; además se puede aplicar a zonas urbanas, suburbanas y rurales lo que convierte al modelo en una aplicación real para minimizar el tiempo en el despliegue de medición inteligente en una ciudad o país.

El trabajo propuesto advierte que no se requiere usar algoritmos de clusterización para agrupar a medidores inteligentes en un punto de agregación de datos debido a la aleatoriedad con la que este tipo de algoritmos trabaja, la metodología definida en un previo árbol de mínima expansión, un setcover y finalmente un enrutamiento usando el algoritmo Dijkstra en su versión desde la estación base celular a los medidores inteligentes mantiene un tiempo de ejecución adecuado para verificar resultados con diferente número de medidores inteligentes.

Con la presente investigación se ha validado el funcionamiento y la confiabilidad de la heurística propuesta en la solución óptima de despliegue de transformadores de potencia para redes de distribución en media y bajo voltaje observando restricciones de capacidad y cobertura. Es un modelo capaz de calcular caídas de voltaje en la red de baja tensión para cada transformador emplazado, garantiza la observancia de las restricciones, lo que hace que, el mapa de ruta obtenido por el modelo sea el óptimo para su respectiva implementación. Además, mediante teoría de grafos y rutas mínimas se garantiza que la topología o mapa de ruta obtenido por el presente modelo sea el mínimo y, por lo tanto, el costo de implementación, de igual manera, sea el mínimo. El modelo, no solo proporciona el mapa de ruta, sino que, proporciona longitud en kilómetros de la ruta que debe seguir el tendido eléctrico, cantidad de pozos o postes, cantidad y capacidad de los transformadores a 
emplazarse con valores estandarizados, ubicación georreferenciada de los dispositivos dispersos en el área de interés, considera además estratificación en base a los consumos tipo y, además, proporciona la capacidad requerida de la subestación en MVAs. Por lo tanto,

el modelo es capaz de dar solución al problema combinatorio de complejidad NP-Complete. Por último, mediante el software Cymdist validamos la funcionalidad del modelo y la capacidad de proporcionar topologías o mapas de ruta óptima para posteriormente mediante el simulador analizar el comportamiento estrictamente eléctrico considerando parámetros eléctricos no tomados en cuenta en la heurística propuesta, tales como, impedancias, coseno phi, configuraciones de subestaciones, etc.

En futuros trabajos implementaremos restricciones de caídas de voltaje para la selección de la ubicación georreferenciada de los transformadores de distribución y, además, se incluirá flujos de potencia en el algoritmo.

\section{Agradecimientos}

Este trabajo ha sido producido gracias al apoyo del GIREI - Grupo de Investigación en Redes Eléctricas Inteligentes de la Universidad Politécnica Salesiana Ecuador bajo el proyecto Planeación y Despliegue Óptimo de Redes de Distribución Eléctrica Aéreas y Soterradas.

\section{Referencias}

[1] E. Inga, S. Céspedes, R. Hincapié, and A. Cárdenas, "Scalable Route Map for Advanced Metering Infrastructure Based on Optimal Routing of Wireless Heterogeneous Networks," IEEE Wirel. Commun., no. April, pp. 1-8, 2017.

[2] M. Campaña, E. Inga, and R. Hincapié, "Optimal placement of universal data aggregation points for smart electric metering based on hybrid wireless," in CEUR Workshop Proceedings, 2017, vol. 1950.

[3] A. Peralta, E. Inga, and R. Hincapié, "Optimal Scalability of FiWi Networks Based on Multistage Stochastic Programming and Policies," J. Opt. Commun. Netw., vol. 9, no. 12, p. 1172, 2017.

[4] F. Aalamifar, G. N. Shirazi, M. Noori, and L. Lampe, "Cost-efficient data aggregation point placement for advanced metering infrastructure," in Smart Grid Communications (SmartGridComm), 2014 IEEE International Conference on, 2014, pp. 344-349. 
[5] G. Rolim, D. Passos, I. Moraes, and C. Albuquerque, "Modelling the Data Aggregator Positioning Problem in Smart Grids," 2015 IEEE Int. Conf. Comput. Inf. Technol. Ubiquitous Comput. Commun. Dependable, Auton. Secur. Comput. Pervasive Intell. Comput., pp. 632-639, 2015.

[6] D. Li, Z. Aung, J. R. Williams, and A. Sanchez, "Efficient Authentication Scheme for Data Aggregation in Smart Grid with Fault Tolerance and Fault Diagnosis," pp. 1-8, 2011.

[7] J. Inga, E. Inga, R. Hincapié, and G. Cristina, "Optimal Planning for Deployment of FiWi Networks based on Hybrid Heuristic Process," Lat. Am. Trans. IEEE (Revista IEEE Am. Lat., vol. 15, no. 9, 2017.

[8] A. Peralta, E. Inga, and R. Hincapié, "FiWi Network Planning for Smart Metering Based on Multistage Stochastic Programming," Lat. Am. Trans. IEEE (Revista IEEE Am. Lat., vol. 13, no. 12, pp. 3838-3843, 2015.

[9] E. Inga and R. Hincapié, "Matched Channel Allocation for Advanced Metering Infrastructure based on Cognitive Mobile Virtual Network Operator," Lat. Am. Trans. IEEE (Revista IEEE Am. Lat., vol. 14, no. 4, pp. 1780-1785, 2016.

[10] G. Rajalingham, Q.-D. Ho, and T. Le-Ngoc, "Evaluation of an efficient Smart Grid communication system at the neighbor area level," in Consumer Communications and Networking Conference (CCNC), 2014 IEEE 11th, 2014, pp. 426-431.

[11] J. Inga, E. Inga, R. Hincapié, and C. Gómez, "Evaluación de la Infraestructura de Medición y la Respuesta de la Demanda," Rev. Técnica Energía, no. 12, pp. 262-269, 2016.

[12] M. Maier, "FiWi access networks: Future research challenges and moonshot perspectives," 2014, pp. 371-375.

[13] E. Inga-Ortega, A. Peralta-Sevilla, R. C. Hincapie, F. Amaya, and I. Tafur Monroy, "Optimal dimensioning of FiWi networks over advanced metering infrastructure for the smart grid," 2015 IEEE PES Innov. Smart Grid Technol. Lat. Am. (ISGT LATAM), pp. 30-35, 2015.

[14] E. Inga-ortega, A. Peralta-Sevilla, R. Hincapié, F. Amaya, and I. Tafur, "Optimal Dimensioning of FiWi Networks over Advanced Metering Infrastructure for the Smart Grid," Innov. Smart Grid Technol. Lat. Am. (ISGT LATAM), 2015 IEEE PES, pp. 30-35, 2015.

[15] A. Peralta-Sevilla, E. Inga, R. Cumbal, and R. Hincapié, "Optimum Deployment of FiWi Networks using Wireless Sensors based on Universal Data Aggregation Points," Commun. Comput. (COLCOM), 2015 IEEE Colomb. Conf., 2015.

[16] A. Alhamwi, W. Medjroubi, T. Vogt, and C. Agert, "GIS-based urban energy systems models and tools: Introducing a model for the optimisation of flexibilisation technologies in urban areas," Appl. Energy, vol. 191, pp. $1-9,2017$.

[17] P. Nageler et al., "Novel validated method for GIS based automated dynamic urban building energy simulations," Energy, vol. 139, pp. 142-154, 2017. 
[18] B. Howard, L. Parshall, J. Thompson, S. Hammer, J. Dickinson, and V. Modi, "Spatial distribution of urban building energy consumption by end use," Energy Build., vol. 45, pp. 141-151, 2012.

[19] H. Li, W. Mao, A. Zhang, and C. Li, "An improved distribution network reconfiguration method based on minimum spanning tree algorithm and heuristic rules," Int. J. Electr. Power Energy Syst., vol. 82, pp. 466-473, 2016.

[20] C. Mateo et al., "European representative electricity distribution networks," Int. J. Electr. Power Energy Syst., vol. 99, no. January, pp. 273-280, 2018.

[21] E. Inga, J. Inga, E. Correa, and R. Hincapié, "Reconstrucción del patrón de consumo eléctrico a partir de Big Data mediante técnica de MapReduce ( Reconstruction of the Electric Consumption Pattern from Big Data using MapReduce Technique )," Enfoque UTE, vol. 9, pp. 177-187, 2018.

[22] J. Inga-ortega, E. Inga-ortega, and C. Gómez, "Electrical Load Curve Reconstruction required for Demand Response using Compressed Sensing Techniques," IEEE PES Innov. Smart Grid Technol. Conf. - Lat. Am. (ISGT Lat. Am., 2017.

[23] G. Davidescu, T. Stutzle, and V. Vyatkin, "Network planning in smart grids via a local search heuristic for spanning forest problems," IEEE Int. Symp. Ind. Electron., pp. 1212-1218, 2017.

[24] N. Funabikiy, K. Uemura, T. Nakanishi, and W. Hassan, "A minimum-delay routing tree algorithm for access-point communications in Wireless Mesh Networks," 2008, pp. 161-166.

[25] B. Karimi, V. Namboodiri, and M. Jadliwala, "On the scalable collection of metering data in smart grids through message concatenation," 2013, pp. 318-323.

[26] J. M. Smith and B. Toppur, "Euclidean Steiner minimal trees, minimum energy configurations, and the embedding problem of weighted graphs in E3," Discret. Appl. Math., vol. 71, no. 1-3, pp. 187-215, Dec. 1996.

[27] B. Krishnamachari, D. Estrin, and S. Wicker, "The Impact of Data Aggregation in Wireless Sensor Networks," Distrib. Comput. Syst. Work. 2002. Proceedings. 22nd Int. Conf., pp. 0-3, 2002.

[28] R. Xie and X. Jia, "Transmission-Efficient Clustering Method for Wireless Sensor Networks Using Compressive Sensing," IEEE Trans. Parallel Distrib. Syst., vol. 25, no. 3, pp. 806-815, Mar. 2014.

[29] I. F. Senturk, K. Akkaya, and F. Senel, "An effective and scalable connectivity restoration heuristic for Mobile Sensor/Actor Networks," in 2012 IEEE Global Communications Conference (GLOBECOM), 2012, pp. 518-523.

[30] O. Kahveci, T. J. Overbye, N. H. Putnam, and A. Soylemezoglu, "Optimization framework for topology design challenges in tactical smart microgrid planning," 2016 IEEE Power Energy Conf. Illinois, PECI 2016, pp. 1-7, 2016.

[31] H. L. Nguyen and U. T. Nguyen, "Bandwidth efficient multicast routing in multi-channel multi-radio wireless mesh networks," in Ultra Modern Telecom- 
munications \& Workshops, 2009. ICUMT '09. International Conference on, 2009, pp. 1-8.

[32] S. Abeysinghe, J. Wu, M. Sooriyabandara, M. Abeysekera, T. Xu, and C. Wang, "Topological properties of medium voltage electricity distribution networks," Appl. Energy, vol. 210, pp. 1101-1112, 2018.

[33] C. Long, J. Wu, L. Thomas, and N. Jenkins, "Optimal operation of soft open points in medium voltage electrical distribution networks with distributed generation," Appl. Energy, vol. 184, pp. 427-437, 2016.

[34] K. G. Boroojeni, M. H. Amini, S. S. Iyengar, M. Rahmani, and P. M. Pardalos, "An economic dispatch algorithm for congestion management of smart power networks: An oblivious routing approach," Energy Syst., vol. 8, no. 3, pp. 643-667, 2017.

[35] M. H. Amini, B. Nabi, and M.-R. Haghifam, "Load Management Using Multi-Agent Systems in Smart Distribution Network," IEEE Power Energy Soc. Gen. Meet., pp. 1-5, 2013.

\section{Sobre los autores}

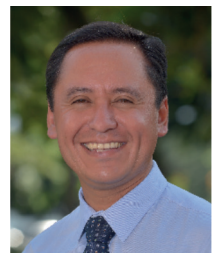

Esteban Mauricio Inga Ortega (Y'1976-M'08). Esteban Inga es investigador científico en la Universidad Politécnica Salesiana Sede Quito. En el año 2001 se gradúa de Ingeniero Electrónico en la Universidad Politécnica Salesiana - Sede Cuenca. En el año 2008 recibió su Maestría en Educación y Desarrollo Social por la Universidad Tecnológica Equinoccial, a inicio del año 2017 recibe su grado de Magíster en Ingeniería por la Universidad Pontificia Bolivariana de Medellín - Colombia y en Noviembre de 2017 obtiene su grado de Ph.D de la Universidad Pontificia Bolivariana de Medellín - Colombia con mención de honor Magna Cum Laude otorgado cuando la tesis supera las expectativas de lo planteado en el proyecto, por concepto unánime de los jurados, y se demuestra producción científica. Es coordinador del grupo de investigaciones en redes eléctricas inteligentes GIREI de la Universidad Politécnica Salesiana, coordinador del programa de Maestría en Electricidad-Sede Quito, miembro activo de IEEE, investigador acreditado Senescyt y miembro del comité científico INEDITA-Senescyt

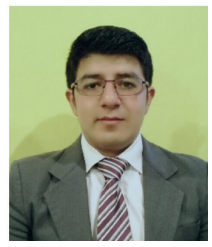

Miguel Campaña (Y'1989-M’05). Se graduó de Ingeniero Eléctrico en la Universidad Politécnica Salesiana-Ecuador, investigador del Grupo de Investigación GIREI. Su trabajo se basa en modelación matemática y técnicas de simulación de redes de distribución eléctrica. Sus intereses de investigación incluyen la asignación de recursos en redes de malla inalámbricas para AMI y el sistema de distribución de eléctrica soterrada y aérea. 